\title{
Recherches opérationnelles sur les soins post-abortum: Progrès et défis. Compte rendu d'une réunion mondiale
}

Dale Huntington

Follow this and additional works at: https://knowledgecommons.popcouncil.org/departments_sbsr-rh How does access to this work benefit you? Let us know!

\section{Recommended Citation}

Huntington, Dale. 1998. "Recherches opérationnelles sur les soins post-abortum: Progrès et défis. Compte rendu d'une réunion mondiale." New York: Population Council. 
Recherches opérationnelles sur les soins post-abortum : Progrès et défis

Compte rendu d'une réunion mondiale 


\section{TABLE DES MATIÈRES}

Liste des graphiques

Remerciements

Introduction

Définition de la notion d'avortement dangereux et de l'ampleur de ce problème de santé publique

Mise en place de nouveaux services post-abortum : conception et mise en œuvre des interventions

Évaluation de la qualité des soins post-abortum

Études de suivi des patientes post-abortum

Étude des coûts des services de soins post-abortum

Décentralisation et élargissement des services de soins post-abortum

Dimensions déontologiques de la recherche opérationnelle sur les soins post-abortum

Orientations futures des recherches opérationnelles sur les soins post-abortum

Documents présentés à la réunion

Liste des participants 
Liste des graphiques

Graphique 1 Chronologie des soins post-abortum

Graphique 2 Cadre pour des soins post-abortum de qualité

Graphique 3 Admission dans le service d'OB/GYN pendant les 30 jours qu'a duré l'étude sur les

$$
\text { hôpitaux du secteur public en Égypte }
$$

Graphique 4 Efficacité des modèles de prestation de services

Graphique 5 Pourcentage de femmes acceptant une méthode de contraception moderne, Bolivie

Graphique 6 Conseils et services fournis en matière de planification familiale postabortum, Pérou

Graphique 7 Douleurs ressenties par les femmes lors des soins post-abortum, Mexico Graphique 8 Cadre pour l'introduction d'améliorations aux programmes de soins postabortum 

Recherches opérationnelles sur les soins post-abortum : Progrès et défis

Compte rendu d'une réunion mondiale

Population Council 
Recherches opérationnelles sur les soins post-abortum : Progrès et défis

Compte rendu d'une réunion mondiale

19-21 janvier $1998 \quad$ New York

Préparé par

Dale Huntington

Membres du Comité de coordination

Coordonnateur de la réunion

Projet RO/AT Afrique

Projet RO/AT ANE

Projet INOPAL

Ipas

Programme Ebert
Dale Huntington

Ian Askew

M. E. Khan, John Townsend

James Foreit

Janie Benson

Karen Stein

Population Council 
Population Council

One, Dag Hammarskjöld Plaza

New York, New York 10017

Tél. : (212) 339-0500

Fax. : (212) 755-6052

E-mail : pubinfo@popcouncil.org

Site Web: www.popcouncil.org

Le Population Council est un organisme international, non gouvernemental, à but non lucratif qui a pour objectif d'améliorer le bien-être et la santé de la reproduction des générations actuelles et futures partout dans le monde et d'arriver à établir un équilibre humain, équitable et durable entre les populations et les ressources. Le Council effectue des recherches dans le secteur biomédical, en sciences sociales et sur la santé publique, tout en contribuant à renforcer les capacités des pays en développement dans le secteur de la recherche. Créé en 1952, le Council est géré par un conseil d'administration international. Son siège de New York appuie un réseau mondial de bureaux régionaux et nationaux.

Les Projets RO/AT en Afrique, en Asie et au Proche-Orient, ainsi que le Projet INOPAL sont financés par le Bureau de la population de l'Agence américaine pour le développement international aux termes des contrats No DPE-3030-C-00-3008, DPE-C-00-90-002-10 et CCP-C-00-95-00007-00 (respectivement).

Copyright (C) 1998 Population Council, Inc.

Toutes les informations figurant dans ce rapport peuvent être reproduites ou adaptées pour répondre à des besoins locaux sans autorisation préalable du Population Council à condition que ces extraits soient distribués gratuitement ou au prix de revient (sans bénéfices), et que la source soit citée. Il faut par contre demander une autorisation préalable au Population Council pour toute reproduction à des fins commerciales. Le Population Council vous serait gré de lui faire parvenir un exemplaire des matériels reprenant des extraits ou des diagrammes figurant dans ce rapport. 


\section{Remerciements}

La Réunion mondiale sur la recherche opérationnelle en matière de soins post-abortum a pu avoir lieu grâce au talent et qualités administratives de plusieurs personnes travaillant dans le domaine des soins de santé de la reproduction pour les femmes. Le comité d'organisation était composé d'Ian Askew, Janie Benson, James Foreit, Dale Huntington, M. E. Khan, Donna Nager et Karen Stein. Une fois l'idée de la réunion lancée, ce groupe a suivi toutes les étapes pour la mener à son terme. L'appui et les conseils constructifs de Sarah Harbison, Marge Horn et Karin Ringheim nous ont évité de nous égarer dans nos réflexions. Plusieurs autres personnes, trop nombreuses pour que nous puissions toutes les citer ici, ont participé à l'organisation logistique de la réunion. Certains de nos collègues méritent toutefois d'être mentionnés, d'autant plus que la réunion qui devait avoir lieu en Égypte a été déplacée à New York : Beth Levine et Donna Nager à New York; Julie Solo à Nairobi; et Sahar Hegazi et Sharon Omar au Caire. Ce compte rendu est le fruit d'une collaboration entre Robert Heidel et Christina Tse du Bureau du Population Council à New York. Janie Benson, Ronnie Johnson, Tim King et Karen Stein, dont les précieux commentaires ont permis au produit final de voir le jour, ont révisé le manuscrit. En plus de la contribution intellectuelle et originale de toutes les personnes que nous avons mentionnées et des auteurs des documents, nous sommes tous convaincus que ce secteur négligé de la santé des femmes mérite d'être amélioré. Espérons que ce rapport atteindra non seulement cet objectif mais encouragera également d'autres personnes à œuvrer en faveur d'une amélioration de la qualité des prestations post-abortum. 


\section{Introduction}

Les femmes qui ont subi des complications à la suite d'un avortement incomplet sont parmi les patientes les plus négligées des soins de santé de la reproduction. Les soins médicaux qui leur sont administrés se limitent couramment à l'utilisation de techniques de dilatation et curetage (D et C) dépassées, le plus souvent sous anesthésie générale. La technique de l'avortement par aspiration n'est pas très répandue dans les pays en développement, bien qu'il ait été prouvé que son utilisation pendant le premier trimestre de la grossesse (sous anesthésie locale) faisait baisser les coûts hospitaliers, réduisait les risques pour la patiente et lui permettait de se rétablir plus rapidement. On accorde trop souvent une attention insuffisante aux autres problèmes de santé de la patiente - par exemple, aux infections de l'appareil génital, aux maladies sexuellement transmissibles et aux fausses couches répétées. Outre la médiocrité généralisée des soins médicaux, la communication entre le personnel soignant et la patiente lors du traitement post-abortum est mauvaise dans la plupart des établissements. Les femmes ne reçoivent généralement pas de conseils sur la procédure médicale à suivre et sur la convalescence post-opératoire. L'orientation vers des services de planification familiale, soit dans le cadre de la visite de suivi à l'hôpital, soit en orientant les femmes vers les cliniques de planification familiale les plus proches, n'est pas systématique au moment de la sortie de l'hôpital.

Le changement d'administration intervenu aux États-Unis en 1992, ainsi que les recommandations de la Conférence internationale sur la population et le développement de 1994 ont aplani les difficultés liées à la recherche sur les questions relatives à l'interruption volontaire de la grossesse et sur l'amélioration des services médicaux post-abortum. Les Projets de recherches opérationnelles et d'assistance technique (RO/AT) du Population Council en Afrique, en Asie et au Proche-Orient (ANE), ainsi qu'en Amérique latine et aux Caraïbes (INOPAL) ont rapidement permis d'occuper ce nouveau champ ouvert à la recherche. Les Projets RO/AT en Afrique, en Amérique latine et dans les Caraïbes ont permis de conclure des accords de sous-traitance avec Ipas, une agence de coopération basée aux Etats-Unis, afin d'entreprendre des études de recherches opérationnelles sur les soins post-abortum dans ces régions. En Afrique francophone, une collaboration s'est établie avec le JHPIEGO. Le Projet RO/AT ANE a coopéré avec le Programme Ebert du Population Council, AVSC International et Ipas à la réalisation d'études en Égypte et en Turquie, alors que le personnel du Population Council en Inde réalisait des études générales sur la disponibilité et la qualité des services post-abortum en se fondant sur les sources d'information existantes. En outre, des recherches opérationnelles post-abortum ont été effectuées par d'autres institutions et organisations multilatérales (telles que le FNUAP et l'Union européenne) coopérant avec l'USAID, ainsi que par des fondations privées.

Une réunion mondiale sur le thème des recherches opérationnelles sur les soins post-abortum : Progrès et défis a été organisée au Bureau du Population Council du 19 au 21 janvier 1998; elle a permis de faire le point sur les progrès réalisés grâce aux trois projets régionaux de recherches opérationnelles sur l'amélioration des prestations de soins post-abortum.Les ressources des trois contrats régionaux de recherches opérationnelles ont été réunies et, en collaboration avec Ipas et le Programme Ebert, environ 65 chercheurs, administrateurs de programmes et représentants gouvernementaux de pays d'Afrique, d'Amérique latine, du Moyen-Orient et d'Asie du Sud-Est se sont réunis. (Veuillez consulter la liste des participants et des auteurs des documents mentionnés plus loin à la fin du rapport.)

Trois objectifs ont été poursuivis lors de la planification de la réunion : 1) examen des progrès accomplis concernant la mise au point de méthodologies de recherches opérationnelles ayant trait aux soins post-abortum; 2) identification des éléments à prendre en considération lors de la mise en place de nouveaux services de soins post-abortum; 3 ) domaines sur lesquels pourraient porter les prochaines recherches opérationnelles sur les soins post-abortum. La réunion a été organisée autour de la présentation de 20 documents rédigés et distribués à tous les participants plusieurs semaines à l'avance. Lors de chaque séance, les participants ont présenté une synthèse des documents et montré la direction que devrait prendre les prochaines études de recherches opérationnelles. Ce rapport est une synthèse des principaux thèmes et points présentés dans ces documents et lors des débats, organisés autour du thème discuté à chacune des séances. Les lecteurs se référeront aux auteurs de chacun des documents pour obtenir des informations complémentaires ou des exemplaires de leurs travaux. 


\section{Définition de la notion d'avortement dangereux et de l'ampleur de ce problème de santé publique}

La première partie de la réunion a porté sur la définition des soins post-abortum, suivie d'une discussion sur le problème de santé publique que représente l'avortement dangereux. Forrest Greenslade a fait un exposé sur le développement des services post-abortum et sur l'introduction de l'avortement par aspiration comme catalyseur de l'évolution des pratiques cliniques (Greenslade et al. $)^{*}$. L'initiative internationale relative aux soins post-abortum, qui aborde la santé de la reproduction des femmes dans le cadre de la santé publique, a pour but de favoriser un transfert rapide de connaissances et de matériels afin de faire baisser les taux de mortalité et de morbidité maternelles liés aux avortements pratiqués dans des conditions dangereuses. Bien que des spécialistes de la santé publique du monde entier travaillent sur la question des avortements dangereux depuis plusieurs décennies, l'évolution de la situation à partir de 1982 (voir graphique 1) révèle que l'adoption du concept de soins post-abortum a eu une forte incidence sur la santé de la reproduction des femmes.

L'élaboration d'une stratégie post-abortum dans le cadre de soins de qualité a visiblement facilité les choses (voir graphique 2). L'adoption de concepts issus du paradigme dominant qui consiste à évaluer les programmes de planification familiale permet aux chercheurs et aux administrateurs de programmes de prendre immédiatement les mesures nécessaires pour améliorer les services médicaux post-abortum. L'introduction d'une nouvelle technologie, l'évacuation par aspiration manuelle, a permis de moderniser les pratiques cliniques relatives au soulagement de la douleur et à la prévention des infections. L'introduction de cette technologie s'accompagne d'une amélioration de la communication entre les personnel soignant et les patientes, essentiellement en matière d'informations et de conseils, attirant ainsi l'attention des prestataires de soins sur les méthodes qui tiennent davantage compte des problèmes des femmes.

L'avortement est une question sensible, surtout lorsque la loi impose des restrictions, ce qui explique pourquoi l'initiative des soins post-abortum continuera à souffrir de certaines limitations. «Il est essentiel que les décideurs comprennent et reconnaissent la nature essentielle du lien qui unit la santé des femmes et les soins post-abortum et qu'ils soient bien conscients que les services de soins post-abortum sont incomplets s'ils ne s'accompagnent pas d'une planification familiale efficace »(Greenslade $e t$ al.).

La première étape permettant d'allouer des ressources aux programmes qui visent à améliorer les services post-abortum consiste à déterminer l'ampleur du problème de santé publique que posent les avortements dangereux. Il est prouvé que l'interruption volontaire de grossesse est l'une des interventions les plus difficiles à analyser même lorsqu'elle est acceptée sur le plan juridique, en raison des perceptions sociales et religieuses largement répandues qui entourent l'avortement. Les évaluations sont difficiles car les avortements ne sont pas toujours déclarés et les analyses secondaires utilisées pour établir les taux d'avortement ne permettent pas vraiment d'étudier la morbidité liée à cette intervention. Les études hospitalières peuvent remplacer les études communautaires, mais cette méthode exige que des données soient réunies systématiquement à des fins d'établissement de statistiques.

Selon Ezzeldin Osman Hassan, le nombre de femmes ayant subi un avortement dans les hôpitaux publics égyptiens est un bon exemple de recherches opérationnelles à des fins d'élaboration de politiques (Huntington et al.). Cette étude, qui est représentative de la situation nationale, a été réalisée sur la base d'échantillons prélevés au hasard dans environ $15 \%$ des hôpitaux du secteur public égyptien. Sur les 22656 admissions dans les services d'obstétrique et de gynécologie pendant la période ininterrompue de 30 jours qu'a duré l'étude, $19 \%$ des patientes venaient se faire traiter pour une interruption volontaire de grossesse ou un avortement spontané (voir graphique 3). Selon les projections, le nombre d'interruptions volontaires de grossesses serait de 14,75 sur 100 grossesses. Dans ce pays, les avortements incomplets sont le plus souvent traités chirurgicalement par la technique de la dilatation et du curetage (D et C) sous anesthésie générale. L'interruption de la grossesse intervenait en moyenne à 10,8 semaines et en grande majorité (86\%) à moins de 12 semaines, ce qui indiquait clairement la nécessité d'introduire des instruments d'évacuation par aspiration manuelle pour le traitement post-abortum en Égypte*

\footnotetext{
* Les références données entre parenthèses se rapportent aux documents présentés lors de la réunion dont la liste se trouve aux pages $\mathrm{xx}-\mathrm{xx}$.

* Les instruments d'évacuation par aspiration manuelle sont plus efficaces lorsque la grossesse est récente et ils sont recommandés pratiquement partout lors du premier trimestre de la grossesse.
} 
On peut recueillir beaucoup d'informations sur l'avortement en examinant les symptômes et les complications dont souffrent les femmes ayant subi un avortement. L'étude réalisée en Égypte révélait que si $14 \%$ des patientes qui arrivaient à l'hôpital perdaient beaucoup de sang, $1 \%$ seulement d'entre elles montraient des signes de traumatisme et $5 \%$ présentaient un ou plusieurs symptômes d'infection. Il semblerait donc que les avortements sont généralement provoqués par des pratiques médicales bénines, par exemple l'utilisation inappropriée d'une contraception d'urgence à la suite de relations sexuelles non protégées.

Il ressort de l'étude réalisée en Égypte que les enquêtes hospitalières peuvent être modifiées afin de réunir des données de bonne qualité qui décriront avec précision les pratiques médicales et les caractéristiques des patientes. L'utilisation de formulaires médicaux abrégés dans les hôpitaux pourrait avantageusement remplacer les recherches communautaires sur l'avortement. En Égypte, les conclusions de cette étude ont semble-t-il été utilisées pour stimuler le débat politique et établir des programmes destinés à améliorer le traitement médical administré aux patientes ayant subi un avortement.

\section{Mise en place de nouveaux services post-abortum : conception et mise en auvre des interventions}

L'approche préconisée par Ipas a largement contribué à améliorer le traitement médical des avortements incomplets depuis le lancement de l'initiative de soins post-abortum (Greenslade $e t$ al.). La méthode générale utilisée dans toutes les études de recherches opérationnelles décrites ici a consisté à préconiser un traitement global de la patiente plutôt que d'améliorer un seul aspect des services (par exemple l'utilisation d'instrument d'évacuation par aspiration manuelle). L'idée est d'améliorer à la fois les compétences techniques grâce à de nouvelles procédures chirurgicales et la communication de toutes les catégories de personnels avec la patiente. L'introduction d'instruments d'évacuation par aspiration manuelle modifie généralement les pratiques cliniques. Dans certains établissements, les soins post-abortum ont été déplacés dans d'autres services. Les documents présentés lors de cette réunion, établis sur la base d'études réalisées en Afrique et en Amérique latine, révèlent des variations régionales concernant la mise en place de services de soins post-abortum améliorés.

Julie Solo et ses collègues (Solo et al.) ont présenté une étude réalisée au Kenya portant sur trois modèles de prestations hospitalières, basés tous les trois sur des procédures globales de prise en charge des soins administrés aux patientes ayant subi un avortement, mais avec une approche différente des services de planification familiale. Dans deux des modèles, les méthodes de contraception sont fournies par le service d'OB/GYN aux patientes hospitalisées. Dans l'un des cas, c'est le personnel du service d'OB/GYN qui fournit la contraception et dans le second cas, c'est le personnel des services ambulatoires de santé maternelle et infantile/planification familiale qui se rend dans le service d'obstétrique et de gynécologie pour fournir aux patientes les méthodes de contraception. Le troisième modèle expérimental porte sur l'orientation des patientes post-abortum du service d'obstétrique et de gynécologie vers la clinique de santé maternelle et infantile/planification familiale de l'hôpital où sont dispensés les services de planification familiale (les patientes qui exprimaient le désir d'obtenir des contraceptifs sont accompagnées à la clinique de planification familiale avant leur départ de l'hôpital). Un large éventail d'indicateurs de succès ont été comparés avant et après le test dans chacun des six hôpitaux étudiés. Ils ont permis d'évaluer la possibilité d'améliorer les services, les coûts associés aux changements, l'acceptabilité des prestations (par les prestataires de soins et les patientes), les changements intervenus dans la qualité des soins fournis et l'efficacité relative des trois modèles concernant l'amélioration du recours à la planification familiale.

Selon Solo et ses collègues, un changement notable de la qualité des services post-abortum a été constaté dans les trois cas (voir graphique 4). Le modèle selon lequel le personnel du service d'obstétrique et de gynécologie responsable des soins post-abortum s'occupe de la planification familiale s'est avéré le plus efficace à plusieurs égards : nombre de femmes conseillées, acceptation de la planification familiale et fourniture des méthodes de contraception. L'ajout des services de planification familiale aux services hospitaliers d'obstétrique et de gynécologie a entraîné un réaménagement des locaux (pour les consultations et le stockage des méthodes de contraception) qui a entraîné des dépenses supplémentaires. Il semble que les patientes qui ont consulté les services de planification familiale de la clinique de santé maternelle et infantile/planification familiale ont été mieux conseillées que celles qui ont consulté le service d'obstétrique et de gynécologie (ce qui indique que le personnel du service hospitalier a besoin d'améliorer ses 
compétences en matière de communication). Cependant, comme un grand nombre de patientes orientées vers cette clinique ne se sont pas présentées pour ce suivi, l'efficacité du modèle est limitée.

L'amélioration des services post-abortum en Afrique francophone est relativement récente si on la compare à l'Afrique de l'Est. L'exposé d'Anita Gosh sur les mesures préliminaires visant à introduire des changements dans le traitement des patientes ayant subi un avortement au Burkina Faso montre bien les problèmes auxquels est confrontée l'Afrique francophone (Gosh et al.). Une série de réunions consultatives a permis de faire des adeptes de l'initiative de soins post-abortum. L'évaluation des services de deux hôpitaux gouvernementaux a révélé la nécessité d'améliorer ces services et de leur fournir un soutien consolidé pour que des changements puissent être introduits. Au Burkina Faso, les services étaient médiocres : ce pays ne s'était pas doté de politiques et de normes nationales concernant le traitement de l'avortement incomplet, les directives ayant trait à la planification familiale ne faisaient pas référence à la contraception post-abortum, les méthodes de prévention des infections laissaient à désirer et les connaissances techniques du personnel sur les complications et le suivi post-abortum étaient incomplètes. Le pays s'est alors doté d'un programme de formation axé sur le renforcement des compétences afin d'introduire des changements dans les protocoles de prise en charge des cas, notamment concernant l'utilisation de la technique d'évacuation par aspiration manuelle et l'offre de services de planification familiale. La nécessité d'améliorer les procédures cliniques d'aseptisation était à l'origine des changements apportés à ce programme. L'attention apportée à l'aseptisation, qui est indispensable dans tout service d'obstétrique et de gynécologie pour garantir un accouchement sans danger, a apparemment facilité l'introduction de changements ayant spécifiquement trait aux soins post-abortum.

En Amérique latine, comme en Afrique, l'avortement est souvent limité par la loi et sanctionné par les convictions religieuses et culturelles. Dans ces pays, les interventions ont porté sur l'amélioration des relations entre les prestataires de soins et les patientes, afin que les patientes soient traitées avec plus de compassion, ainsi que sur l'amélioration des procédures chirurgicales et d'autres aspects du traitement médical. L'exposé de Cecilia Garcia Barrios sur les travaux menés dans un grand hôpital public d'Oaxaca, au Mexique, a révélé des variations régionales concernant l'application de l'initiative mondiale de soins post-abortum (Langer et al.).

Oaxaca est la capitale d'un État rural pauvre du Mexique. L'hôpital mentionné dans cette étude traite un ou deux avortements par jour, chez des patientes qui parlent souvent l'une des 20 langues locales ou un dialecte et qui ne comprennent pas l'espagnol (la langue parlée par les médecins). Cette étude quasi expérimentale conçue pour tester les améliorations apportées aux prestations de services a été réalisée en réunissant des données sur un large éventail d'indicateurs de la qualité des soins. En outre, des entretiens en profondeur avec le personnel et les patientes ont permis d'évaluer leur perception des changements apportés aux services post-abortum. Les principales mesures prises dans le cadre de l'intervention étaient les suivantes : 1) modification des pratiques hospitalières afin de réduire l'attente, d'améliorer la prise en charge de la douleur et de respecter l'intimité des femmes; 2) passage de la technique de la dilatation et du curetage à celle de l'évacuation par aspiration manuelle; 3 ) amélioration des relations entre les patientes et les prestataires de services, en accordant une attention particulière aux conseils sur la contraception postabortum; 4) production de matériels rédigés spécialement pour accompagner les conseils. Les mesures prises dans le cadre de l'étude d'Oaxaca pour améliorer la communication inter-personnelle, notamment celles visant à supprimer les obstacles linguistiques et sociaux entre les prestataires et les patientes appartenant à des minorités ethniques, sont un exemple original d'interventions visant à modifier les comportements lors des prestations de soins post-abortum.

Les résultats de l'étude réalisée à Oaxaca révèlent des changements positifs dans le traitement des patientes post-abortum. L'attente, en particulier post-intervention, avait fortement diminué. Les instruments permettant de pratiquer l'évacuation par aspiration manuelle ont également été acceptés immédiatement : avant l'étude, $10 \%$ seulement des cas étaient traités de cette manière, alors que le taux était passé à $78 \%$ des cas pour lesquels cette méthode était appropriée après l'intervention. Les résultats concernant la prise en charge de la douleur étaient mitigés. Bien que les prestataires de services aient effectivement commencé à remplacer plus systématiquement l'anesthésie générale par une anesthésie locale après la réalisation de l'étude, les mesures cliniques visant à soulager la douleur avant et après l'intervention n'avaient pas réellement changé, bien que l'accent ait été mis à plusieurs reprises sur ces mesures pendant la formation et, par la suite, durant les visites de suivi des superviseurs. 
Grâce aux efforts visant à améliorer la communication inter-personnelle, les patientes de l'hôpital étaient mieux traitées et elles étaient mieux et plus fréquemment informées. Par exemple, «en général, les médecins et les infirmières ont commencé à faire attention en installant la patiente sur la table d'opération et lui ont expliqué plus gentiment comment se placer» (Langer et al., p. 17). L'amélioration de la communication inter-personnelle a notamment entrainé un accroissement notable du pourcentage de femmes ayant accepté d'utiliser une méthode de contraception après un avortement (de 29 à $58 \%$ après l'étude) et du pourcentage de femmes qui ont obtenu cette méthode avant de quitter l'hôpital (qui est passé de 29 à $57 \%$ ). Les auteurs estiment toutefois que malgré ces résultats remarquables, les relations entre les prestataires de services et les patientes étaient toujours entachées d'une certaine méfiance et d'un peu de crainte. Il faudra encore déployer de grands efforts et faire preuve de beaucoup de constance pour modifier des comportements dictés par des convictions culturelles lors des prestations de soins médicaux. L'originalité de l'étude d'Oaxaca est d'avoir abordé ces problèmes.

L'influence du milieu médical sur les services fournis aux patientes post-abortum a également été mis en lumière par la deuxième étude réalisée en Amérique latine qui a été discutée lors de cette réunion. Juan Diaz a expliqué que les sanctions juridiques appliquées en cas d'avortement avaient eu des conséquences négatives sur les soins administrés aux patientes post-abortum dans trois hôpitaux boliviens du secteur public. Les enquêteurs ont identifié plusieurs problèmes; par exemple les patientes devaient systématiquement répondre à des questions destinées à identifier les cas d'avortement illégal; les tarifs étaient plus élevés pour les patientes présentant des signes d'avortement provoqué (des prix prohibitifs pour de nombreuses femmes, ce qui les pousse à attendre avant de chercher de l'aide) et les prestatires de services appliquaient des pratiques liées à des protocoles de prise en charge des cas contraires aux normes. Le Ministère bolivien de la santé a reconnu les faits et demandé au Population Council de mener une étude pilote afin d'identifier les améliorations possibles des pratiques cliniques qui permettraient d'améliorer la qualité des soins et l'utilisation de la planification familiale par les patientes qui avaient avorté.

Contrairement à toutes les autres études de recherches opérationnelles sur les soins post-abortum examinées lors de la réunion, la Bolivie n'a pas abandonné la pratique chirurgicale du curetage en faveur de l'évacuation par aspiration manuelle. Le changement a consisté à déplacer ce service dans une salle refaite à neuf. On a privilégié une prise en charge globale de la patiente, en mettant l'accent sur la communication inter-personnelle et sur l'orientation vers des services compétents (notamment pour l'obtention de méthodes de contraception). On a constaté une amélioration sensible des compétences techniques des prestataires de services en matière de traitement post-abortum et de l'utilisation de la contraception à la suite d'un avortement (voir graphique 5). Grâce à l'amélioration de ses compétences techniques, le personnel a pu conseiller plus fréquemment les patientes et leur fournir des informations plus complètes. L'importance accordée aux relations inter-personnelles dans le programme de formation n'a pas été inutile : il semble que le personnel montre davantage de compassion et accorde plus d'attention aux femmes. En outre, grâce à l'amélioration des compétences en matière de communication, les adolescentes ont commencé à fréquenter les hôpitaux qui avaient participé à l'étude lorsque les communautés des alentours ont su que la qualité des soins s'était améliorée. Les patientes post-abortum ont également commencé à mieux accepter la planification familiale : le taux d'utilisation qui était pratiquement nul avant l'étude est passé à $46 \%$ dans l'un des hôpitaux et même à 81 \% dans un autre hôpital, grâce aux liens établis entre les soins améliorés et la planification familiale.

Les auteurs notent que si plusieurs études pilotes ont permis d'améliorer la qualité des soins postabortum en Amérique latine, les projets sont rarement élargis et les résultats peu durables. Ils en ont conclu que «l'inefficacité de la mise en ouvre de ces programmes avait une origine sociale et politique plutôt que technique» (Diaz et al., p. 13). Les trois facteurs entravant la mise en place de services post-abortum intégrés dans le cadre du programme de soins de santé de la reproduction et de planification familiale relevés dans cette étude sont les suivants : a) des obstacles juridiques et administratifs qui obligent les femmes à pratiquer des avortements clandestins quand les prestations de santé de la reproduction sont inexistantes; b) la faiblesse du statut socio-économique des femmes et les restrictions apportées à l'avortement, qui font que le gouvernement n'accorde qu'une faible priorité aux besoins sanitaires des femmes; c) l'influence de longue date de la politique suivie par Mexico qui, récemment encore, représentait une force restrictive en Amérique latine. L'amélioration de l'accès aux services de traitements des complications post-abortum fut une première étape importante qui a permis de surmonter certains des 
obstacles que nous avons décrits, en particulier en permettant aux femmes de se faire soigner plus rapidement, ce qui a réduit les risques pour leur santé.

Khama Rogo, qui a présenté les documents de cette séance, a souligné l'importance des approches communautaires qui permettent de créer de nouveaux services et de limiter les risques liés aux avortements dangereux. En raison de l'importance accordée à l'aspect clinique de l'initiative de soins post-abortum, la conception des nouveaux services a bénéficié d'une attention limitée; voilà pourquoi les approches non hospitalières et du secteur privé n'ont pas été examinées en profondeur. Il conviendrait notamment d'étudier le rôle du secteur privé dans les futurs programmes de soins post-abortum. L'initiative pour une maternité sans risques comportait des interventions à la fois cliniques et communautaires dans tous ces volets excepté celui des soins post-abortum; à l'avenir, il faudra donc examiner les prestations offertes dans des établissements autres que les grands hôpitaux du secteur public. 


\section{Évaluation de la qualité des soins post-abortum}

La cohérence géographique sur des questions telles que la conception des études et les méthodes d'évaluation est garantie par le personnel des Projets de recherches opérationnelles depuis la Conférence internationale sur la population et le développement de 1994. Les premières études sur les soins postabortum réalisées en Turquie et en Égypte ${ }^{*}$ ont permis d'établir des directives sur les indicateurs et les modèles d'instruments de collecte de données, qui ont été adaptées aux études suivantes. La standardisation des indicateurs de soins post-abortum a été facilitée par la concordance dans la conception des études, des méthodes de collecte de données, et par l'amélioration des soins étudiés dans le chapitre précédent. Par exemple, le recours à des recherches pré- et post-tests a donné aux enquêteurs l'idée de préparer des questionnaires à faire remplir aux patientes au moment de la sortie de l'hôpital. Le regroupement des pratiques cliniques et des relations personnelles autour de l'introduction de la technique d'évacuation par aspiration manuelle a permis de faire un lien entre le choix des indicateurs et l'intervention standard permettant d'améliorer les services. Le projet DATAPAC exécuté par Ipas et INOPAL permettra aux chercheurs du monde entier de mettre au point un ensemble d'instruments de base. Bien que les résultats des études présentés à la réunion de janvier révèlent une standardisation progressive de l'évaluation de la qualité des services post-abortum, ils font également apparaître des variations spécifiquement nationales.

L'étude réalisée au Pérou par Janie Benson et ses collègues sur un modèle de soins intégrés postabortum dans un hôpital public (Benson et al.) est un exemple des méthodes les plus sophistiquées utilisées actuellement pour évaluer la qualité des services post-abortum. On savait que ces services étaient trop coûteux et de qualité médiocre. Le Ministère péruvien de la santé a pris note de ces défauts et a demandé qu'une étude de recherches opérationnelles soit réalisée sur l'incidence de l'introduction de services postabortum intégrés sur les pratiques cliniques et sur l'utilisation des ressources. L'introduction d'un modèle intégré de services post-abortum entraînait des modifications de la structure hospitalière, notamment : a) des modifications des protocoles de prise en charge du traitement post-abortum, b) une réduction des tarifs appliqués aux patientes traitées dans ces services, c) une modification du type de fournitures, d'équipements et de services utilisés dans les services post-abortum (entraînant des modifications des mécanismes hospitaliers d'appui logistique), d) une nouvelle définition des emplois compte tenu des nouvelles responsabilités du personnel, e) plusieurs améliorations de l'infrastructure hospitalière dans laquelle les patientes sont traitées.

Les modifications apportées aux prestations de services post-abortum ont entraîné des améliorations réelles, qui ont pu être déterminées grâce à un cadre d'évaluation de la qualité des soins* . Ce modèle permet de réunir des données sur les indicateurs de compétences techniques des prestataires de soins et sur la communication entre le personnel et les patientes pendant l'hospitalisation. Par exemple, les premières conclusions de cette étude révèlent que l'utilisation de la technique d'évacuation par aspiration manuelle a progressé, passant de 0 à $91 \%$ pour les patientes pour lesquelles elle convient. La proportion de patientes satisfaites de la qualité des soins prodigués a également progressé à la suite de l'intervention (voir figure 6). Par exemple, la proportion de patientes ayant reçu des informations sur le diagnostic de leur cas est passée de $11 \%$ avant l'étude à $28 \%$ après l'étude, l'augmentation étant de 10 à $46 \%$ pour les informations sur leur traitement et de 18 à $79 \%$ sur les méthodes de planification familiale. La durée du séjour à l'hôpital des patientes post-abortum a diminué, passant d'un peu plus de 33 heures à environ 6 heures. Lorsque l'administration a compris qu'elle entrainait une modification des ressources utilisées pendant le séjour hospitalier, elle a diminué de moitié le montant des frais à la charge des patientes. Les auteurs de l'étude estiment que l'appui des principaux responsables de l'administration était indispensable au succès de ce programme pilote. La production de résultats liés à un cadre de qualité des soins notoires fut un élément critique pour obtenir un appui permettant d'améliorer les prestations.

Dans certaines études, notamment le modèle intégré de services post-abortum appliqué au Pérou et dans d'autres pays, la qualité des services a pu être améliorée relativement rapidement. L'introduction de la

\footnotetext{
* Dale Huntington et al., 1996. «The quality of abortion services in Turkey», International Journal of Gynecology and Obstetrics 53 : 41-44; Dale Huntington et al., 1995. «Improving the medical care in counseling of postabortion patients in Egypt», Studies in Family Planning 26, No 6 :350-362.

" Judith Bruce,1990. «Fundamental elements of the quality of care: A simple framework», Studies in Family Planning 21, No 2 : 61-91.
} 
méthode d'évacuation par aspiration manuelle avec administration réduite d'analgésiques, alliée à des améliorations de la qualité des conseils et des autres pratiques cliniques, a permis de mieux renseigner les patientes et d'accroître l'utilisation de la planification familiale. Bien que la plupart des études aient révélé que des mesures avaient été prises pour soulager la douleur chez les patientes post-abortum, cet aspect de la qualité des services reste difficile à gérer car certaines femmes affirment encore avoir ressenti de fortes douleurs. La perception de la douleur est très difficile à évaluer car elle est influencée par divers stimuli, tant environnementaux que physiologiques.

Jaime Fuentez Velasquez et ses collègues ont étudié la douleur ressentie par les femmes après un avortement dans le cadre d'une enquête plus large comparant trois modèles de soins post-abortum appliqués dans six grands hôpitaux de la ville de Mexico (Fuentez et al.). Dans le premier cas, l'évacuation par aspiration manuelle s'accompagne d'une anesthésie paracervicale, de l'administration d'analgésiques ou de sédatifs pour contrôler la douleur et de conseils à la patiente pendant toute la durée du traitement. Dans le deuxième et dans le troisième modèle, l'hôpital utilise la méthode de la dilatation et du curetage accompagnée de l'administration de sédatifs, de somnifères et d'analgésiques puissants pour lutter contre la douleur, rendant la patiente pratiquement inconsciente. Dans le deuxième modèle, la patiente reçoit également des conseils, bien qu'elle soit inconsciente pendant les périodes au cours desquelles elle est sous l'emprise de sédatifs puissants. Le troisième modèle est le service actuel, sans amélioration du traitement ni conseils (voir figure 7). Les enquêteurs ont interrogé les femmes sur le type de douleurs ressenties, l'endroit où elles se manifestaient et leur intensité, ainsi que sur leur état émotionnel. En outre, une technique «d'observation du comportement pendant la douleur» a été utilisée pour enregistrer des manifestations physiques codées de la douleur (telles que les expressions du visage, les signes vitaux, la position du corps, etc.) avant, pendant et après le traitement; un dernier entretien a eu lieu immédiatement avant la sortie de l'hôpital afin d'évaluer la douleur ressentie à ce moment-là.

Les résultats préliminaires des entretiens avec 144 femmes ont révélé que la majorité d'entre elles ressentaient des douleurs légères à modérées avant l'intervention. Au moment de la sortie de l'hôpital, toutes les femmes ressentaient des douleurs, surtout celles à qui l'on avait administré des sédatifs puissants (modèle 3), alors que celles qui avaient subi une évacuation par aspiration manuelle sous anesthésie locale, accompagnée de bons conseils ressentaient les douleurs les moins violentes (modèle 1). L'évaluation de l'état émotionnel des patientes sur la base de leurs déclarations portait essentiellement sur les aspects négatifs : nervosité, inquiétude, la tristesse et dépression - une technique d'évaluation qui n'encourageait pas les femmes à faire des commentaires sur des aspects plus positifs de leur état psychologique, tels que le sentiment de soulagement et la confiance en soi. La formation des prestataires de soins sur la manière d'aider les femmes sur le plan émotionnel s'est généralement avérée positive. Concernant la douleur que les femmes s'attendaient à ressentir, il ressort de l'étude que dans le cas de l'évacuation par aspiration manuelle sous anesthésie locale, les femmes déclaraient généralement avoir ressenti davantage de douleurs que ce qu'avaient noté les observateurs, et que les patientes ayant subi un curetage déclaraient plus fréquemment avoir ressenti une douleur violente accompagnée d'une sensation de brûlure. Les patientes pensaient toutes avoir reçu davantage d'analgésiques qu'on ne leur en avait en fait administré.

Il ressort de l'étude que bien que toutes les patientes post-abortum aient déclaré avoir ressenti des douleurs pendant les soins, la souffrance était la plus forte avant l'intervention. On pourrait soulager partiellement la douleur en donnant une formation aux prestataires de soins leur permettant de conseiller et d'apporter un soutien psychologique aux patientes. Même si l'administration de sédatifs puissants supprime toute sensation de douleur pendant l'intervention, les patientes à qui l'on avait administré des doses plus faibles de sédatifs déclaraient généralement avoir ressenti moins de douleurs après l'intervention. Il ressort de l'étude réalisée au Mexique que beaucoup de patientes post-abortum souffrent encore inutilement. Les résultats indiquent clairement qu'il faut améliorer la formation des prestataires de services à une utilisation plus efficace des analgésiques lors des soins post-abortum.

Les exposés sur les études de recherches opérationnelles mesurant la qualité des soins postabortum ont ensuite porté sur l'évaluation des systèmes médicaux en vigueur. On passa alors à l'examen des études d'Analyse Situationnelle portant sur les services pratiquant les avortements en Inde et en Turquie. Dans les deux cas, les études ont été réalisées en utilisant des instruments basés sur la méthodologie d'Analyse Situationnelle mise au point par le Population Council pour évaluer la qualité des services 
d'interruption volontaire de la grossesse. L'avortement est légal dans ces deux pays, bien que soumis à des restrictions légèrement différentes.

L'interruption médicale de la grossesse a été légalisée en Inde en 1972 en cas de problèmes sociaux et médicaux. Ces services étaient surtout disponibles dans les centres de santé communautaire et les hôpitaux ruraux; récemment, quelques centres de santé primaire ont commencé à offrir ces services. La majorité des 6,7 millions d'avortements qu'affiche l'Inde chaque année sont pratiqués dans des établissements qui ne sont pas reconnus officiellement, par un personnel non compétent, dans de mauvaises conditions d'hygiène. Bien que les statistiques nationales révèlent une augmentation de $46 \%$ des interruptions médicales de la grossesse pendant la décennie 1981/82-1991/92, le taux s'est stabilisé en 1985 avec environ 600000 interruptions médicales de la grossesse par an, ce qui représente $10 \%$ du nombre total d'avortements affiché pour l'ensemble du pays. Le nombre moyen d'interruptions médicales de la grossesse pratiqué dans chaque clinique officielle a diminué, passant de 111 en 1981/82 à 89 en 1991/92. M. E. Khan et ses collègues ont présenté une analyse des indicateurs de la qualité des services d'interruption médicale de la grossesse dans l'ensemble du système afin de permettre au Gouvernement de prendre les mesures nécessaires pour enrayer le recul affiché par le secteur public dans le cadre de sa nouvelle directive en faveur de la santé de la reproduction et de l'enfant (Khan et al.).

Une Analyse Situationnelle des services d'avortement a été réalisée dans 751 cliniques situées dans les États de Gujarat, Maharashtra, Uttar Pradesh et Tamil-Nadu (regroupant ensemble environ $37 \%$ de la population indienne). L'échantillon a révélé une répartition inégale des établissements offrant des services d'interruption médicale de la grossesse et des disparités profondes en matière de fréquentation des cliniques officielles. Les données non scientifiques recueillies auprès de plus de 500 médecins révèlent une augmentation des cas post-abortum en raison du nombre croissant de femmes qui se font avorter dans des établissements non officiels. Il ressort de l'étude que les cliniques censées fournir des services d'interruption médicale de la grossesse ne fonctionnaient pas toutes : entre 16 et $32 \%$ des centres de santé primaire certifiés n'avaient jamais offert ce service en raison du manque de personnel compétent et d'équipements, ce qui révèle des lacunes dans le système de formation et la difficulté d'assurer une formation adéquate lorsque le nombre de cas est relativement faible. Malgré la faiblesse des liens avec les services de planification familiale en Uttar Pradesh et au Maharashtra et leur relâchement dans de nombreux établissements, dans l'État du Tamil-Nadu, l'interruption médicale de la grossesse était liée à l'acceptation préalable d'une méthode de planification familiale, en particulier de la stérilisation. Généralement, l'intégration entre l'interruption médicale de grossesse et une planification familiale appropriée et éthique faisant défaut, ce volet du programme doit être amélioré.

Levent Cagatay (Pile et al.) a révélé une deuxième application de la méthodologie d'Analyse Situationnelle en Turquie. Dans ce pays, l'interruption volontaire de grossesse a été légalisée en 1983 pour les femmes mariées, à condition qu'elle soit pratiquée avant la dixième semaine, avec le consentement du mari. Avant l'annulation des restrictions légales imposées à l'avortement, on a constaté une augmentation régulière des taux d'avortement dans les années 60 et 70, entraînant une augmentation des taux de mortalité maternelle provoquée par des complications liées à l'avortement. La communauté médicale turque n'a pourtant pas appuyé la légalisation de l'avortement, probablement en raison des profits qu'elle tirait des avortements illicites. Bien que ces incitations économiques aient été limitées par la légalisation de l'avortement, elles ont continué à entraver les efforts visant à établir des liens entre les services de planification familiale et les services d'avortement. Cet aspect est particulièrement important étant donné que la méthode de planification familiale la plus populaire en Turquie reste le retrait, responsable de près des deux tiers des grossesses se soldant par un avortement. Dans ce contexte, il n'est pas surprenant qu'en 1993, une grossesse sur cinq se soit soldée par une interruption volontaire de la grossesse et qu'entre 28 et $40 \%$ des femmes turques aient eu au moins un avortement provoqué.

En 1994, une Analyse Situationnelle des services d'avortement a été réalisée dans 16 grands hôpitaux situés dans sept provinces turques. Ces établissements pratiquaient $48 \%$ du nombre total d'avortements déclarés par le Ministère turc de la santé en 1994. Les méthodes de collecte de données ont porté sur une liste intégrée d'indicateurs de la qualité des soins : un inventaire de tous les équipements, fournitures et matériels disponibles; des entretiens avec le personnel et les patientes; et des observations standardisées de toutes les interventions. Les résultats ont révélé les points forts et les lacunes du programme turc. 
L'étude révèle que les médecins généralistes pratiquent près de la moitié des avortements, bien qu'en tant que groupe, ils ne représentent qu'un tiers du nombre total de prestataires de soins capables d'assurer cette intervention. Les obstétriciens et les gynécologues exercent plus rarement dans le secteur public, qu'ils abandonnent aux généralistes, car ils préfèrent pratiquer l'intervention dans leur cabinet privé. La disponibilité des services d'avortement est encore limitée par le nombre d'établissements assurant ce service. Dans l'ensemble du pays, «27 \% seulement des hôpitaux du secteur public assurent des services d'avortement et deux centres de santé maternelle et infantile/planification familiale pratiquent des avortements», bien qu'en théorie, tous les hôpitaux généraux et tous les centres de santé maternelle et infantile/planification familiale gouvernementaux soient autorisés à assurer ces services (Pile et al., p. 10).

Les résultats du test de connaissances factuelles que l'on a fait passer aux prestataires de soins et l'observation des interventions ont révélé que les médecins des établissements du secteur public avaient de bonnes connaissances des techniques chirurgicales d'avortement, pratiquant l'aspiration sous vide, mais que leurs autres connaissances (évaluation et dépistage médicaux, conseils, mesures de prévention de l'infection) étaient faibles, voir inexistantes. L'administration d'analgésiques était beaucoup trop rare en raison notamment des protocoles de prise en charge des cas qui déconseille l'anesthésie générale (en raison des risques pour la santé) sans attirer l'attention sur les autres types de médicaments analgésiques administrables. Il est ressorti de l'étude que $48 \%$ des patientes venues se faire avorter n'avaient reçu aucun médicament contre la douleur pendant l'intervention; de ce fait, $37 \%$ des patientes ont déclaré avoir ressenti des douleurs extrêmememt violentes et encore $46 \%$ des femmes, des douleurs modérées. En raison du manque d'enthousiasme à assurer des prestations de planification familiale (les avortements répétés étant une source de revenus pour les spécialistes) les conclusions de l'étude concernant les liens avec le programme de planification familiale ne sont pas vraiment surprenantes. Le taux d'utilisation de la planification familiale chez les femmes turques ayant subi un avortement était faible. Bien que $84 \%$ des avortements aient été pratiqués dans des établissements capables de fournir des méthodes de planification familiale, $40 \%$ seulement des patientes ont quitté l'établissement en possession d'une méthode de contraception.

Lorsque Ian Askew a passé en revue les documents présentés lors de la session, il a constaté que les indicateurs d'évaluation de la qualité des soins étaient pratiquement tous identiques. Il s'est demandé si cette concordance pouvait s'expliquer par la pertinence universelle des indicateurs ou par les liens qui se sont établis entre les chercheurs et il a demandé que des efforts supplémentaires soient déployés afin d'identifier d'autres indicateurs permettant d'élargir notre cadre conceptuel. Plusieurs possibilités prometteuses sont apparues, notamment le besoin urgent d'évaluer la place qu'occupe la qualité des soins dans les préoccupations des patientes. Les nouvelles recherches devraient permettre d'enquêter sur la perception qu'ont les femmes de l'avortement et des soins post-abortum*. Il faut également s'assurer que le choix des indicateurs répond aux besoins des administrateurs de programmes. Par exemple, bien que les travaux portent généralement sur des indicateurs établis au niveau de l'établissement que l'on peut regrouper pour les appliquer aux programmes (comme c'est le cas pour les études d'Analyse Situationnelle), les résultats ne pourront être utilisés dans le cadre de la planification et de l'évaluation des programmes que si les recherches opérationnelles sont dotées d'un plan permettant de les diffuser et de les intégrer aux activités. Askew a conclu son exposé en plaidant en faveur d'études capables de mesurer l'impact de différents niveaux de qualité sur les comportements à plus long terme en matière de santé de la reproduction, notamment sur la réduction de la morbidité liée aux avortements incomplets.

\section{Études de suivi des patientes post-abortum}

Les études longitudinales de suivi des patientes post-abortum posent plusieurs problèmes spécifiques et se heurtent également aux difficultés classiques des études par sondage. L'obligation éthique de protéger le droit à l'intimité de la patiente post-abortum est un obstacle difficile à surmonter lors des études de suivi, d'autant plus que dans bien des cas la femme comme le prestataire de soins sont exposés à des sanctions juridiques et sociales si l'on découvre que l'avortement a été provoqué. Les règles de conduite

\footnotetext{
* Dale Huntington, Laila Nawar et Dallia Abdel -Hedy ont commencé à s'intéresser à ce problème en 1997. «Women's perceptions of abortion in Egypt», Reproductive Health Matters 9 (mai).
} 
culturelles et sociales empêche encore la patiente qui a subi un avortement de révéler la cause de son hospitalisation. La patiente post-abortum court donc un risque potentiel élevé d'être exposée à des sanctions physiques et psychologiques si elle participe à une étude de suivi. Ces considérations, auxquelles vient s'ajouter l'attention accrue portée à la protection de la confidentialité et au consentement préalable en connaissance de cause, limitent encore la précision des entretiens de suivi.

Ronnie Johnson (Johnson et al.) a parlé d'une étude prospective à grande échelle des patientes ayant subi un avortement au Zimbabwe. Les auteurs ont évoqué divers aspects de la conception et de la conduite des travaux qui dévoilent les problèmes qui se posent lors des études de suivi. Les expériences sur le terrain au Zimbabwe révèlent notamment l'effet des taux d'abandon.

Au Zimbabwe, 1776 patientes ont été enrôlées sur les sites d'intervention de l'étude et 1114 patientes sur les sites du groupe de comparaison. Sur les sites de l'intervention, les patientes ont été enrôlées dans l'étude après amélioration des services post-abortum grâce à l'application d'un grand programme de formation. Ainsi, l'étude de suivi mesure l'impact sur les patientes des changements intervenus dans la qualité des soins, notamment en ce qui concerne leur utilisation de la planification familiale. Des taux d'acceptation (pour l'entretien de suivi) de, respectivement, $72 \%$ et $57 \%$ (pour chacun des deux groupes étudiés) ont été établis lors des entretiens de recrutement. Ces femmes ont accepté de retourner à l'hôpital au bout de 3, 6,9 ou 12 mois (selon le niveau de l'étude à laquelle elles participaient national ou provincial; il n'y a pas eu d'entretien de suivi au niveau du district). Pour ces visites, il était prévu de rembourser le prix du transport et de verser à ces femmes une petite allocation de repas (les enquêteurs ont organisé des entretiens à domicile pour les patientes qui ne retournaient pas à l'hôpital pour l'entretien). Les premières données ont révélé qu'au bout de trois mois, le taux moyen d'abandon était d'environ $58 \%$ pour les quatre sites participants à l'étude; au bout de six mois, ce taux atteignait $63 \%$ de l'échantillon original, bien que la proportion de femmes ayant abandonné entre le troisième et le sixième mois n'ait été que de $14 \%$. Le nombre de patientes participant toujours à l'étude au bout de six mois était relativement faible (l'un des sites était passé de 120 à 27 femmes au bout de six mois). Si le taux d'abandon était élevé au départ, la proportion de femmes abandonnant l'étude a baissé rapidement par la suite. Johnson et les coauteurs de l'étude estiment que le taux global de participation est relativement bon et que l'étude donnera de bons résultats lorsqu'elle s'achèvera à la fin de 1998 .

Nahla Abdel-Tawab et ses collègues (Abdel-Tawab et al.) ont présenté une deuxième étude de suivi, réalisée en Égypte. Cette étude a examiné l'impact de la participation des conjoints sur la guérison des patientes ayant subi un avortement et sur l'utilisation de méthodes de contraception, tout en respectant le droit des patientes à l'intimité. Un groupe de contrôle expérimental après test a été utilisé pour évaluer l'impact de l'intervention sur le niveau de participation des conjoints et sur les patientes un mois après leur sortie de l'hôpital. Les maris appartenant au groupe d'intervention ont été informés sur divers aspects de la santé de leurs femmes (sur la base d'une recherche formative réalisée antérieurement en Égypte) : la nécessité de se reposer et d'avoir une bonne nutrition, les signes précurseurs, le retour de la fécondité et la nécessité d'utiliser des méthodes de planification familiale. L'impact de ces informations sur le soutien du conjoint (matériel et émotionnel), la guérison physique et émotionnelle de la patiente et sur l'utilisation de la contraception dans le couple a été évalué grâce à des entretiens à domicile avec les patientes et dans le cadre de discussions de groupe avec quelques maris.

Peut-être parce que la période de suivi était plus courte et que les entretiens ont eu lieu à domicile, le taux d'abandon de l'étude égyptienne était plus faible que celui de l'étude zimbabwéenne : $80 \%$ des 366 patientes qui ont accepté de participer à l'étude ont eu un entretien à domicile. Il a été relativement facile de parler aux conjoints, une fois obtenu le consentement de la patiente. La majorité des maris ont accompagné leur femme à l'hôpital ou étaient présents au moment de la sortie. Les conseils ont de toute évidence eu des effets positifs sur le comportement des maris et la guérison des patientes. Par exemple, la détresse provoquée par un avortement incomplet était moins profonde chez les patientes dont les maris avaient été conseillés que chez les autres. Les enquêteurs en ont conclu que les conseils aux maris devraient faire partie intégrale des programmes de soins post-abortum et que les procédures de consentement préalable en connaissance de cause devaient être appliquées strictement afin de protéger le droit de la patiente à l'intimité. 
Emma Ottolenghi, qui participait à cette séance, a souligné qu'il était important d'étudier l'impact à moyen et à long terme des améliorations apportées aux services post-abortum. Le fait que les études réalisées en Égypte et au Zimbabwe aient pu surmonter les obstacles inhérents aux études de suivi s'explique partiellement par la qualité supérieure des soins administrés aux femmes, ce qui les a peut-être encouragées à respecter leurs engagements. Les études soulignent la nécessité d'inclure dans le suivi un large éventail d'indicateurs de résultats qui sont liés de façon démontrable aux interventions expérimentales. Il ressort par exemple d'une étude réalisée au Zimbabwe que l'intervention sur la planification familiale semble avoir été plus concluante dans le domaine de la fourniture des méthodes de contraception que dans la transmission d'informations. Lors de l'évaluation de l'intervention visant à améliorer les aptitudes à donner des conseils, il faudrait avoir recours à des techniques d'observation directe de la qualité de l'interaction; en outre, les entretiens de suivi devraient permettre de vérifier quelles informations ont été retenues et dans quelle mesure les comportements et les attitudes en matière de santé de la reproduction ont changé.

\section{Étude des coûts des services de soins post-abortum}

La rentabilité de l'initiative de soins post-abortum a déjà été examinée * Ces études sur les coûts nous apprennent qu'il est possible de faire des économies importantes grâce à la technique de l'évacuation par aspiration manuelle et d'améliorer la rentabilité grâce à la réduction de la durée du séjour à l'hôpital, à une utilisation moins intensive des ressources hospitalières (matériels et fournitures) et à une utilisation plus efficace du temps du personnel. Ipas, en collaboration avec le projet INOPAL du Population Council, a peaufiné la méthodologie d'évaluation du traitement des patientes ayant subi un avortement utilisée lors des premières études; deux applications de la méthodologie d'étude des coûts ont été présentées à la réunion de janvier.

La méthodologie d'évaluation des coûts de l'agence de coopération Ipas s'appuie sur la comptabilisation des ressources utilisées et l'estimation du coût par patiente. Sur la base d'une étude approfondie des dossiers financiers, administratifs et du personnel de l'établissement, on procède à des estimations des dépenses au titre des fournitures médicales, des salaires et des coûts directs fixes pertinents. L'estimation des frais généraux permet de déterminer le coût moyen du lit par minute et par jour. L'observation porte également sur les patientes ayant subi un avortement afin d'évaluer les coûts variables associés à leur traitement. Pour réaliser une étude du temps et du mouvement, il faut avoir recours à des observateurs qualifiés qui utiliseront des listes de vérification standard et y consigneront le temps passé en contact direct avec chacune des patientes par le personnel médical et non médical de chaque service ou salle de traitements, et le type de fournitures et de médicaments utilisés pour traiter ces patientes. En combinant les informations tirées de l'examen des dossiers administratifs de l'hôpital et de l'étude sur le temps et le mouvement, on peut estimer le coût moyen du traitement post-abortum par unité de temps (généralement calculé par minute et par lit) ou le coût moyen par patiente traitée dans l'établissement. On compare les coûts avant et après avoir procédé au changement du protocole de prise en charge des patientes postabortum, afin d'estimer l'impact des changements sur les dépenses de l'établissement.

Tim King (King et al.) a présenté les études de recherches opérationnelles portant sur les coûts des soins post-abortum en Afrique et en Amérique latine. L'examen des 21 études réalisées dans six pays (neuf au Mexique et sept au Kenya) sur la base de la méthodologie d'étude des coûts mise au point par Ipas, a permis d'établir des comparaisons sur la durée moyenne du séjour à l'hôpital et le montant des coûts liés à l'évacuation par aspiration manuelle par rapport au curetage. On a généralement constaté que le passage à l'évacuation par aspiration manuelle et la réorganisation des services permettaient de réduire d'environ 30 \% la durée du séjour à l'hôpital et de $61 \%$ le montant total des coûts du traitement pour chaque établissement. Une analyse des études comparables en termes de conception et de méthode de collecte de données a révélé que l'utilisation de l'évacuation par aspiration manuelle permettait de réduire d'environ $49 \%$ la durée du séjour et de $23 \%$ les coûts du traitement. Les économies attribuées à l'amélioration des prestations de services post-abortum s'expliquent surtout par la réduction de la durée du séjour (qui correspond à une diminution du temps consacré aux patientes par le personnel et des frais généraux associés à chaque hospitalisation). King et ses collègues ont constaté que si la réduction de la durée du séjour

\footnotetext{
* Voir par exemple Brooke Johnson et al. 1993 «Costs and resources utilized for the treatment of incomplete abortion in Kenya and Mexico», Social Science and Medicine 36, No 11.
} 
s'expliquait partiellement par la réduction de la période de convalescence qui suit une anesthésie locale, la réorganisation des services qui, d'hospitaliers sont devenus ambulatoires dans la majorité des études, a aussi largement contribué à limiter la durée moyenne du séjour.

Carlos Brambila (Brambila et al.) a parlé en détail d'une application de la méthodologie d'étude des coûts de l'agence de coopération Ipas à un programme en cours à Oaxaca, au Mexique, destiné à améliorer les services post-abortum dans un grand hôpital du service public. Les améliorations apportées aux services post-abortum de l'hôpital général d'Oaxaca sont semblables à celles apportées dans d'autres hôpitaux. L'intervention a permis d'introduire des instruments pour procéder à l'évacuation par aspiration manuelle, de privilégier les procédures de conseil et d'établir des liens avec les services hospitaliers de planification familiale grâce à des discussions avec le personnel. L'impact de ces améliorations sur la qualité des soins fournis aux patientes ayant subi un avortement est comparable à celui observé dans les autres établissements. Par exemple, l'utilisation de la méthode d'évacuation par aspiration manuelle est passée de 13 à $65 \%$ des cas. La communication avec les patientes sur le traitement, la convalescence et la planification familiale est devenue plus fréquente et l'éventail des questions discutées s'est élargi. Grâce à l'amélioration des conseils et à l'intégration des services, la prévalence de la contraception chez les patientes ayant quitté l'hôpital est passée de 28 à $44 \%$.

Les économies associées à ces améliorations sont également impressionnantes. Le montant total du coût par patiente a diminué de $32 \%$, passant de 265 à 180 dollars (y compris les coûts associés à l'introduction de ces améliorations). Les économies les plus importantes avaient trait aux instruments, aux médicaments et aux fournitures; les coûts ont été réduits de près de deux tiers, passant de 92 dollars avant l'étude à 32 dollars après amélioration des services. L'autre diminution des coûts portait sur le temps d'utilisation des lits, dont le coût est passé de 150 à 85 dollars. Cette économie est directement attribuable à la réduction de la durée du séjour intervenue pendant l'étude (qui est passé d'environ 30 heures par patiente avant l'étude à 20 heures après l'intervention).

Le temps consacré par le personnel aux différentes étapes des soins, analysé dans le cadre de l'étude des coûts réalisée à Oaxaca (Mexique), a contribué à la mise au point de la méthodologie de l'agence de coopération Ipas. La ventilation du temps consacré aux patientes par le personnel donnait une indication sur le temps nécessaire pour administrer les soins dans chaque service. Cette analyse a révélé qu'avant l'intervention, le temps consacré par le personnel à la patiente était de $49 \%$ plus élevé qu'après l'intervention, ce qui confirmait que l'amélioration des prestations de services avait permis au personnel d'utiliser son temps plus efficacement. Dans ce même ordre d'idée, l'étude révélait que si le temps consacré à la patiente avait diminué, le temps passé avec elle par différentes catégories de personnel variait profondément. Par exemple, après l'intervention, les médecins consacraient davantage de temps au diagnostic par ultrasons mais passaient moins de temps avec les patientes alitées en attente d'un traitement. De même, après l'adoption de la méthode de l'évacuation par aspiration manuelle, les infirmières passaient proportionnellement plus temps avec la patiente que les médecins : le temps consacré par le médecin à la procédure avait diminué de 24 minutes par patiente alors que le temps consacré par l'infirmière avait augmenté d'environ 10 minutes. Si l'économie de temps est faible comparée aux autres économies réalisées, l'analyse détaillée révèle que le temps est utilisé plus efficacement et que le personnel spécialisé est moins présent pendant les soins qui ne sont pas liés au diagnostic ou à la thérapeutique.

Laila Nawar, sur la base d'une étude réalisée en Égypte (Nawar et al.), a donné un autre exemple de variation analytique de la méthodologie de l'agence Ipas. Tout comme pour l'étude réalisée à Oaxaca, l'analyse des coûts présentée par Nawar faisait partie d'un programme plus large destiné à améliorer les soins médicaux post-abortum fournis aux patientes en Égypte. Deux des 10 hôpitaux faisant partie du programme d'amélioration des soins post-abortum ont été choisis pour cette analyse des coûts. Ces deux hôpitaux sont représentatifs des établissements du Ministère égyptien de la santé et de la population, l'un d'eux étant un hôpital général de taille moyenne, l'autre un hôpital de district plus petit. Dans ces deux établissements, les améliorations étaient comparables à celles de l'étude d'Oaxaca. Avant la réalisation des études de recherches opérationnelles post-abortum, le niveau de la qualité des services assurés par ces deux hôpitaux était très faible. Par exemple, environ deux tiers des patientes post-abortum avaient subi un curetage sans anesthésie à l'hôpital de district et les procédures aseptiques n'étaient respectées régulièrement dans aucun des deux établissements. 
Sur la base de la méthodologie établie par Ipas, Nawar et ses collègues ont constaté un accroissement de $32 \%$ des dépenses par patientes post-abortum dans l'hôpital de district et de $8 \%$ dans l'hôpital général (où les canules d'évacuation par aspiration manuelle sont jetées après chaque usage, selon la politique gouvernementale); une utilisation multiple des canules permet de réduire les coûts d'environ $6 \%$, mais cette économie hypothétique a toutefois été compensée par l'augmentation des dépenses liées aux analgésiques et aux fournitures antiseptiques, l'intervention n'ayant pas réduit de manière notable le temps du personnel. L'étude fait également état d'une réduction de la durée du séjour à l'hôpital (69 \% de réduction dans l'hôpital de district et 30 \% dans l'hôpital général) mais les économies réalisées sur les coûts directs fixes associés à la réduction de la durée du séjour hospitalier n'ont pas compensé l'augmentation des dépenses liées aux fournitures, matériel et médicaments. Il ressort de l'étude des coûts réalisée en Égypte que dans les établissements où la qualité des soins post-abortum est inférieure aux normes, les améliorations ont un prix.

Les modifications des coûts absolus associés à l'amélioration des services ne révèlent pas les économies potentielles lorsqu'ils sont analysés en comparaison avec des indicateurs d'amélioration de la qualité des soins. Selon Nawar et ses collègues, les mesures de la rentabilité des services post-abortum peuvent donner des résultats très différents selon l'indicateur de résultat choisi et les caractéristiques de chaque établissement. Par exemple, bien que l'efficacité des interventions destinées à informer davantage de femmes sur la planification familiale ait été prouvée et ait entraîné une économie de $10 \%$, l'hôpital général a affiché une économie de $21 \%$ alors que dans l'hôpital de district, le coût par patiente a augmenté. L'intervention a prouvé sa rentabilité dans les deux cas en matière d'informations sur les consultations de suivi.

La présentation par Andy Thompson de trois études des coûts a entraîné une discussion animée sur la mise au point de cette méthodologie spécialisée de recherches opérationnelles. Les économies issues de la modification des traitements post-abortum entrent dans la catégorie des «économies d'opportunité» (qui consistent à libérer des ressources à utiliser ailleurs) par opposition aux diminutions des coûts qui pourraient entraîner une réduction générale des dépenses hospitalières. Il s'est avéré nécessaire de procéder à d'autres études des coûts post-abortum pour voir comment les ressources libérées grâce à la réduction du séjour hospitalier peuvent être réaffectées et pour savoir si le type de dépenses étudié est véritablement le plus important aux yeux de la direction (les dépenses dont elle est responsable). Par exemple, les économies réalisées sur les cas simples permettent-elles d'affecter davantage de ressources au traitement des patientes souffrant de complications graves? Si les études de coûts post-abortum permettent de répondre à ce type de question, les données ainsi produites auront une incidence directe sur l'administration des services médicaux et pourront être utilisées lors de l'élaboration de politiques relatives aux soins médicaux. 


\section{Décentralisation et élargissement des services de soins post-abortum}

Les hôpitaux du secteur public, dans lesquels les soins sont administrés par des équipes de médecins et d'infirmières, sont le type d'établissements décrits dans les études le plus fréquemment choisi pour améliorer les soins post-abortum. Cependant, les soins post-abortum basés sur la méthode de l'évacuation par aspiration manuelle sous anesthésie locale peuvent parfaitement être administrés par un personnel compétent dans des établissements de soins de santé primaire. Suivant la voie ouverte par l'initiative de maternité sans risques, qui s'appuie sur des sages-femmes et du personnel paramédical spécialisé, les études contemporaines sur les soins post-abortum ont permis d'explorer d'autres stratégies de prestation de services permettant de prodiguer des soins d'urgence accessibles et sans danger.

Dans son exposé sur la décentralisation des services post-abortum dans les zones rurales du Ghana, Victor Ankrah a parlé de la possibilité de confier aux sages-femmes communautaires la responsabilité d'administrer les soins post-abortum (Ankrah et al.). Bien que dans ce pays, la loi permette au personnel médical des établissements certifiés de provoquer des avortements sous certaines conditions, nombre de femmes souffrent des conséquences d'un avortement dangereux. Il a été largement démontré qu'au Ghana les avortements pratiqués dans des conditions dangereuses sont l'une des causes principales de la mortalité liée à la grossesse et que les services d'urgence sont pratiquement inexistants dans les zones rurales. Le Ministère ghanéen de la santé a abordé la question de l'accessibilité et de la sécurité des services postabortum dans un document intitulé : National Reproductive Health Service Policy and Standards, publié en 1996. Lors de la mise en œuvre de cette politique, un volet expérimental a permis de donner une formation sur les soins post-abortum à près de 6000 sages-femmes communautaires dans l'ensemble du pays.

Les contrôles effectués dans le cadre de l'étude avant et après le test ont permis d'examiner les améliorations apportées à la qualité des soins après avoir donné une formation aux sages-femmes communautaires sur les soins post-abortum. Les prestataires de services des hôpitaux locaux (qui supervisent les sages-femmes communautaires) et les sages-femmes ont été interrogés, ainsi que les femmes se présentant dans l'un des six hôpitaux de district à la suite de complications liées à l'avortement. Après collecte des données de base, 40 sages-femmes et quatre médecins ont reçu ensemble une formation sur l'amélioration des procédures de prise en charge des cas. La formation des sages-femmes et des médecins qui les supervisent a eu plusieurs aspects positifs, notamment la mise en place d'un système de supervision basé sur la confiance et l'appui aux services post-abortum décentralisés.

Il ressort de l'étude réalisée au Ghana sur les sages-femmes que le recours au personnel paramédical vivant dans les communautés améliore considérablement l'accès aux services post-abortum : l'attente avant le traitement pour les femmes qui s'adressaient aux sages-femmes était d'environ 90 minutes, alors qu'elle était d'environ 220 minutes pour les femmes qui s'étaient présentées à l'hôpital de district. Le coût du traitement administré par les sages-femmes était moins élevé et les patientes ont affirmé qu'elles faisaient confiance aux sages-femmes et qu'elles les jugeaient compétentes. Celles-ci ont utilisé avec succès la méthode de l'évacuation par aspiration, sans complications, et tous les instruments ont été utilisés selon des protocoles d'aseptisation stricts. Les sages-femmes se sont non seulement montrées capables de suivre des protocoles de prise en charge des cas, mais également d'assurer les consultations de suivi de leurs patientes, notamment sur la planification familiale post-abortum. Il ressort de l'étude que la collaboration avec les hôpitaux de district (où travaillent les superviseurs) a permis aux sages-femmes d'améliorer leurs compétences professionnelles dans d'autres secteurs. En outre, comme elles vivent à proximité des communautés où elles travaillent et comprennent parfaitement les problèmes des femmes, plusieurs de ces sages-femmes ont organisé des activités d'éducation communautaire afin de sensibiliser les femmes et les hommes au problème des avortements dangereux et des grossesses non désirées.

Deborah Billings a donné un bon exemple de recours aux accoucheuses traditionnelles dans le cadre des soins post-abortum sur la base des résultats d'une étude ethnographique réalisée dans la région rurale de Morelos, au Mexique (Billings et al.). Comme au Ghana, la mortalité maternelle s'explique en grande partie par les complications liées aux avortements pratiqués dans des conditions dangereuses : selon des statistiques provenant des établissements du secteur public, les complications liées à l'avortement représentent la troisième ou quatrième cause de mortalité maternelle au Mexique, ce qui s'explique en grande partie par le fait que les femmes rurales ont un accès limité aux services d'urgence. Malgré l'attention accordée par le Gouvernement mexicain à l'amélioration des compétences des accoucheuses 
traditionnelles, avant cette étude de recherches opérationnelles, on n'avait jamais tenté de donner une formation sur les soins post-abortum à de tels prestataires de soins. Billings et ses collègues ont réalisé une étude qualitative de terrain sur les concepts et expériences des accoucheuses traditionnelles ayant trait à l'avortement et ils ont comparé les connaissances des sages-femmes avant et après leur participation à un programme de formation sur les soins post-abortum portant essentiellement sur la stabilisation de tous les types de complications et sur l'orientation des patientes ayant besoin d'un traitement post-abortum.

Il ressortait des premières recherches que les connaissances des sages-femmes sur les complications liées à l'avortement étaient incomplètes et imprécises. En outre, bien que la formation ait renforcé leurs connaissances sur certains problèmes médicaux, les croyances populaires concernant les facteurs provoquant une fausse couche (passage à côté d'un cimetière, lavage des châles) persistaient généralement. Le but de la formation post-abortum de ces accoucheuses traditionnelles était de compléter le traitement qu'elles administraient et de décourager les croyances dangereuses. Les accoucheuses traditionnelles ont donc des rituels pénétrés de symbolisme, elles prescrivent des remèdes à base de plantes et utilisent la physiothérapie, intégrant ces méthodes au système médical moderne qui s'appuie sur les signes précurseurs du danger et les protocoles d'orientation. Billings et ses collègues ont fait remarquer qu'il convenait d'utiliser des modèles différents pour former et évaluer les sages-femmes que ceux utilisés pour instruire le personnel hospitalier. L'intégration des croyances populaires et de la pharmacopée autochtone au cadre conceptuel occidental des signes de danger et des soins de suivi doit être mieux comprise pour que le recours aux accoucheuses traditionnelles soit plus efficace.

D'autre part, les sanctions normatives appliquées lors des interruptions volontaires de la grossesse dans les communautés de l'État de Morelos ont limité l'efficacité des accoucheuses traditionnelles en tant que prestataires de soins post-abortum. Ces obstacles ont notamment entravé la mise en place d'un réseau d'orientation vers l'hôpital le plus proche pour le traitement rapide des avortements provoqués incomplets. La crainte des accoucheuses d'être traitées «d'avorteuses» aurait empêché certaines d'entre elles d'administrer un traitement médical d'urgence. En outre, les sages-femmes ont eu le sentiment d'être rejetées et condamnées par les médecins des hôpitaux modernes (dans lesquels le personnel n'était pas toujours conscient du rôle joué par les sages-femmes et ne les soutenait pas) lorsqu'elles accompagnaient des femmes ayant besoin de soins d'urgence. Ces sentiments et ces expériences rendent encore plus difficile la création d'un réseau d'aiguillage viable des avortements incomplets dans l'État de Morelos. L'organisation d'ateliers communs pour les sages-femmes et les médecins, comme l'a fait le Ghana, est un moyen d'abaisser les barrières qui séparent les systèmes de soins traditionnels et modernes.

Achola Ominde (French et al.) a donné un troisième exemple d'amélioration de l'accès aux services post-abortum en s'appuyant sur une nouvelle catégorie de personnel sanitaire. Afin d'accroître le nombre d'établissements offrant des services post-abortum (méthode de l'évacuation par aspiration manuelle), le Ministère kényan de la santé a eu recours à la recherche opérationnelle pour examiner la possibilité d'introduire des services améliorés dans les centres de soins de santé primaires et de district. Pour décentraliser les services, il fallait d'abord former le personnel clinique (paramédical et de niveau intermédiaire dont le rôle est surtout d'assister les médecins mais qui peut occuper des postes de responsabilité) au traitement post-abortum et notamment à l'utilisation de la méthode d'évacuation par aspiration manuelle. Dans les hôpitaux plus importants, le personnel clinique assure généralement les services ambulatoires, notamment la prise en charge des cas post-abortum. Avant la réalisation de l'étude de recherche opérationnelle par Ominde et ses collègues, il n'existait pas de directive autorisant le personnel clinique à fournir des soins post-abortum dans des établissements où il n'y avait pas de spécialiste pour les épauler.

Un membre du personnel clinique et une infirmière de chaque établissement ont reçu une formation leur permettant d'assurer des services post-abortum en équipe. On a évalué l'attitude du corps médical concernant l'opportunité de confier le traitement post-abortum au personnel médical de niveau intermédiaire en interrogeant des cadres du Ministère de la santé, des représentants d'associations professionnelles, tout le personnel clinique, ainsi que des médecins et des infirmières travaillant dans les trois hôpitaux de district avant et deux mois après le programme de formation. Toutes les personnes interrogées ont estimé que le personnel clinique avait prouvé qu'il était capable d'assurer des services d'évacuation par aspiration manuelle de qualité pour les cas d'avortements incomplets ne présentant pas de complications. Par exemple, le corps médical et les décideurs ont estimé à $86 \%$ après l'intervention (contre 
48\% avant) que la procédure d'évacuation utérine était dans les cordes du personnel clinique. Les hôpitaux tertiaires ont affiché un recul des cas post-abortum s'expliquant par l'augmentation du nombre de cas traités dans les hôpitaux de district et les centres de santé primaire. En outre, le nombre de lits occupés par des patientes post-abortum avait diminué, tout comme l'utilisation de médicaments et de fournitures dans les salles d'opération des établissements de soins tertiaires lorsque les cas post-abortum simples ont été pris en charge au niveau du district et des soins de santé primaire. Sur la base de cette étude, le Ministère de la santé envisage de recommander une modification de la politique gouvernementale afin de garantir au personnel clinique la protection nécessaire pour pratiquer légalement l'évacuation par aspiration manuelle. La logistique permettant de fournir les équipements nécessaires pour appliquer cette procédure à des niveaux inférieurs du système de soins de santé est la prochaine étape dans la décentralisation des services post-abortum au Kenya.

L'élargissement des services post-abortum évoqué dans les études pilotes est apparemment la prochaine étape dans l'évolution des soins post-abortum. Les chercheurs tout comme les administrateurs de programmes commencent à comprendre les problèmes qui s'opposent à l'élargissement des études de recherches opérationnelles post-abortum alors que s'achève la première série d'études organisées au lendemain de la Conférence internationale sur la population et le développement. Dale Huntington a évoqué l'expérience de l'Égypte, où une série d'études de recherches opérationnelles post-abortum a été réalisée, en commençant par une petite étude pilote en 1993, suivie par une étude plus importante et par d'autres recherches sur les politiques (Huntington et Nawar).

Deux types de conditions entourant la recherche opérationnelle sur les soins post-abortum ont des implications profondes sur le mouvement permettant de passer de la recherche opérationnelle à des programmes de soins de santé complets. Le premier a trait à la nature sensible de l'avortement, en particulier dans des circonstances où l'accès aux services est limité sur le plan juridique et sanctionné sur le plan social. En limitant les problèmes étudiés au domaine strictement médical, le concept de soins postabortum a largement contribué à la santé de la reproduction des femmes. Cependant, les soins post-abortum sont entachés d'idées préconçues qui entravent les travaux de leurs partisans tant au sein des gouvernements que dans la communauté médicale.

Le deuxième problème concerne les instruments d'évacuation par aspiration manuelle. Les dons sont un mécanisme expéditif permettant de réaliser des études pilotes ou même de procéder à un élargissement à petite échelle. Mais il n'est pas réaliste d'élargir des services de soins post-abortum tributaires de dons, en particulier d'articles que l'Agence américaine pour le développement international ne peut fournir. Les chercheurs et les administrateurs de programmes doivent s'assurer que les établissements disposent à toutes les étapes du programme des instruments nécessaires pour procéder à l'évacuation par aspiration manuelle. Les chercheurs ne comprennent pas parfaitement les difficultés associées au passage des articles fournis par des donateurs aux articles disponibles dans le commerce, dans le cadre de programmes post-abortum.

Huntington et ses collègues ont constaté que dans une société égyptienne essentiellement conservatrice, ni l'élargissement des services post-abortum, ni d'autres innovations apportées à ce secteur de la population ne feront jamais beaucoup d'adeptes. Ils estiment que la situation sera identique dans des pays en butte à des restrictions légales et sociales similaires sur l'avortement. Il faut alors adopter une approche pragmatique permettant de faire progresser l'objectif d'institutionnalisation. L'établissement de partenariats avec d'autres programmes de maternité sans risques, l'établissement de liens avec des programmes de planification familiale post-partum et l'élargissement constant de groupes bien informés dans la communauté médicale sont indispensables pour que les services puissent prendre de l'ampleur.

Sally Girvin et Amy Pollack, évoquant les recherches opérationnelles sur la décentralisation et sur l'élargissement des études de cas post-abortum, ont souligné qu'il s'agissait de deux initiatives programmatiques distinctes. La décentralisation est envisageable lorsqu'il existe une autorité indépendante du pouvoir central capable de gérer et d'assurer les services. L'élargissement est un processus impliquant l'intégration de changements profonds à une infrastructure existante. Le problème de l'accès aux services de soins post-abortum et de l'amélioration de cet accès peut être abordé en décentralisant les services ou en élargissant les études pilotes de recherches opérationnelles. L'essentiel dans les deux cas est que cet accès 
accru s'inscrive dans un contexte incorporant explicitement tous les éléments qui garantissent ou contribuent à améliorer la qualité des soins prodigués aux patientes.

Les éléments à prendre en compte lorsqu'on prévoit soit de décentraliser soit d'élargir une étude pilote sortent toutefois du cadre de la qualité des soins, celle-ci n'étant en effet qu'un volet de l'ensemble des prestation de services. L'adaptation par Girvin et Pollack de l'approche stratégique de l'OMS concernant la contraception (voir graphique 8) montre bien quels sont les éléments dont il faut tenir compte lors de l'introduction de services post-abortum élargis. La nature interactive des trois volets des prestations de services - perspective de la cliente, système de services et technologie - est une dynamique fondamentale des services de soins. L'interface entre le système de services et la cliente comprend les attitudes du prestataire de services, un aspect critique de la fourniture des services à la lumière du milieu politique, socioculturel et économique entourant les soins post-abortum.

La démarche de l'OMS visant à mieux comprendre les questions liées à l'élargissement des services de soins de santé attire l'attention sur les problèmes entourant la décentralisation des services postabortum. Dans les programmes, il faut faire la distinction entre les initiatives visant à décentraliser une technologie (évacuation par aspiration manuelle) et celles ayant pour but de créer des centres autonomes, dirigés par une autorité locale, assurant des prestations sanitaires. La capacité de définir des politiques régissant les coûts, les livraisons de matériel, le personnel et les systèmes de gestion favorisant les services contrôlés localement, indique que le programme est décentralisé. Les études de recherches opérationnelles post-abortum portant sur les aspects de la décentralisation doivent aussi accorder de l'attention à ces questions et ne pas s'en tenir aux mesures prises pour élargir la diffusion de la technologie de l'évacuation par aspiration manuelle. De même, l'élargissement d'une étude pilote post-abortum ne se limite pas simplement à augmenter le nombre de points de prestation de services équipés de cette technologie. Les services les plus efficaces sont ceux dans lesquels on a intégré les conclusions des recherches réalisées dans des milieux plus complexes, notamment celles des projets de démonstration. En conclusion, Girvin et Pollack ont encouragé les professionnels de la santé à aborder les questions relatives à la technique de l'évacuation par aspiration manuelle dans la perspective des droits fondamentaux et à éliminer les stigmates qui collent aux services post-abortum, afin que les femmes aient accès à des soins de santé de la reproduction sans danger et plus humains.

\section{Dimensions déontologiques de la recherche opérationnelle sur les soins post-abortum}

La conduite éthique de recherches opérationnelles ayant trait aux soins de santé de la reproduction des femmes est un aspect essentiel du travail de terrain. La déclaration d'Helsinki de 1964 donne des directives claires selon lesquelles les intérêts de la science doivent être subordonnés au bien-être des sujets de la recherche. Le consensus sur la nature des normes déontologiques a évolué et englobe aujourd'hui les trois principes fondamentaux suivants : respect de la personne, notamment le droit des individus qui en sont capables à l'autodétermination et la protection des personnes dont l'autonomie est limitée ou diminuée; l'intention bénéfique, qui implique l'obligation déontologique de maximiser les avantages et de minimiser les inconvénients et englobe aussi la notion d'absence d'intentions malveillantes; et la justice pour les sujets de la recherche, en les traitant tous sur un pied d'égalité. Karin Ringheim a passé en revue ces considérations fondamentales dans le contexte de la recherche opérationnelle post-abortum et elle a posé un certain nombre de questions qui ont été examinées dans le cadre des discussions de groupe qui ont suivi son exposé (Ringheim).

Lors d'une table ronde sur l'éthique, la population et la santé de la reproduction, organisée par Columbia University (New York) et le FNUAP à la veille de la Conférence internationale sur la population et le développement de 1994, les participants ont examiné la Déclaration d'Helsinki à la lumière des recherches contemporaines sur la santé de la reproduction et ils ont rédigé une «déclaration de principes déontologiques» regroupant les principes mentionnés ci-dessus et établissant des normes élevées régissant le comportement des chercheurs. Dans son document, qui a le mérite d'inciter à la réflexion, Ringheim passe en revue ces principes dans le cadre de la recherche sur les soins post-abortum.

La difficulté, en matière de recherche opérationnelle, est de s'assurer que des directives déontologiques strictes, semblables à celles définies par Ringheim, sont respectées tant par ceux qui orchestrent les entretiens que par les prestataires de services. Le respect de la confidentialité et du consentement donné en connaissance de cause n'est pas toujours mentionné dans les programmes de 
formation et ne guide pas systématiquement le choix des chercheurs. Il n'existe pratiquement pas de directives sur la formation déontologique des enquêteurs et des prestataires de soins en matière de recherches opérationnelles sur la santé de la reproduction. Plusieurs questions sont en suspens et ne sont pas abordées dans les documents. Par exemple, le consentement en connaissance de cause n'est pas aussi aisé à cerner qu'il y paraît. La terminologie occidentale, fortement axée sur l'individu, qui décrit le droit de se retirer d'une étude, de refuser de répondre à des questions, etc. n'est pas facile à adapter à des traditions culturelles non occidentales. Il faut expliquer à chaque fois la signification de ces déclarations, ainsi que les procédures à suivre, que les personnes interrogées doivent comprendre et accepter. Une supervision et un suivi étroit des interactions entre les clients et les prestataires est nécessaire pour s'assurer que les enquêteurs et les prestataires de services appliquent des procédés éthiques et n'abusent pas de leur pouvoir pour influencer le choix de leurs clients.

Le traitement éthique que les femmes sont en droit d'attendre des prestataires de services est un domaine encore inexploré de la recherche opérationnelle, qui s'est limitée au respect de la déontologie dans les activités de collecte de données. Ringheim se pose la question dans son document de savoir «comment changer des attitudes paternalistes vis-à-vis des femmes selon lesquelles le prestataire de services est convaincu depuis sa naissance d'en savoir plus que sa cliente? Et plus particulièrement en ce qui concerne les soins post-abortum, comment éviter hostilité et négligence à l'égard des clientes dictées par l'attitude négative des prestataires à l'égard de l'avortement?» Ces questions prennent toute leur importance lors de l'administration d'analgésiques, au regard de l'obligation déontologique qui consiste à permettre aux patientes d'exercer un contrôle sur leur traitement et de la responsabilité des prestataires de s'assurer que les soins n'entraînent pas d'inconfort physique ou mental, ne provoquent pas de douleurs ou ne comportent pas de danger. Il ressort de plusieurs études de recherches opérationnelles que le soulagement de la douleur ou l'absence de traitement contre douleur est une question de déontologie que les enquêteurs sur les soins post-abortum se doivent de soulever. En effet, les groupes de discussion qui ont suivi la présentation de Ringheim ont confirmé que l'administration d'analgésiques relevait davantage des prestations de services que de la méthodologie de la recherche.

Le cas particulier de la recherche opérationnelle post-abortum impliquant des femmes célibataires ou des adolescentes, que l'on rencontre souvent, pose plusieurs problèmes déontologiques. Il est parfois difficile de convaincre les prestataires de services locaux que ces femmes doivent participer à l'étude ou, si elles y participent, qu'elles ont droit au respect de leurs droits (consentement, confidentialité) au même titre que les femmes mariées plus âgées. Il faudrait accorder davantage d'attention à ces considérations déontologiques lorsqu'on traite avec ces sujets qui sont plus exposés aux sanctions physiques et psychologiques si leur grossesse ou l'avortement est dévoilé aux membres de la famille. Dans certaines situations, les adolescentes préféreront se faire soigner dans le secteur privé où la confidentialité est mieux protégée. Comme nous l'avons déjà fait remarquer, les recherches opérationnelles post-abortum n'ont généralement englobé les prestations de services hors du secteur public. L'étude des services post-abortum destinés aux adolescentes met donc les chercheurs au défi d'élargir leur études à de nouveaux systèmes de prestations de services et d'établir des normes déontologiques élevées, tant pour les prestataires de services que pour les responsables de la collecte de données.

\section{Orientations futures des recherches opérationnelles sur les soins post-abortum}

D'autres groupes de discussion ont examiné les questions relatives à l'avenir de la recherche opérationnelle sur les soins post-abortum. Janie Benson a résumé la situation et proposé de nouvelles directions (Benson). Les thèmes traités dans son document ont été débattus en groupe. Depuis que les restrictions imposées aux recherches sur l'avortement ont été levées à Mexico, l'utilité des études sur des problèmes post-abortum n'a plus été contestée. Les recherches opérationnelles ont permis de diagnostiquer les obstacles cliniques et autres qui s'opposent à la qualité des soins de santé de la reproduction,

d'influencer les politiques et de tester d'autres modèles de prestations de services. Bien qu'on ait accumulé beaucoup de connaissances récemment, certains domaines sont encore inexplorés et pourraient faire l'objet de nouvelles recherches sur les soins post-abortum.

La plupart des études de recherches opérationnelles ont porté sur des hôpitaux, généralement situés dans des zones urbaines. Ces services étant de plus en plus souvent déplacés vers les centres de santé et de soins primaires, il est essentiel de savoir quelles sont les qualifications dont aura besoin le personnel 
paramédical qui assurera ces services à la périphérie. Plusieurs études présentées à cette réunion ont abordé cette question, mais comme on a tendance à décentraliser les services de soins partout dans le monde, il faut réunir davantage de données d'expérience pour que les administrateurs de programmes sachent vraiment s'y prendre pour élargir ces services aux zones rurales et en faire bénéficier les pauvres des zones urbaines. Le rôle du secteur privé dans l'élargissement des services post-abortum n'a pas non plus été suffisamment exploré.

La perception qu'ont les femmes de l'avortement incomplet et de la grossesse non désirée est une autre question qui a été négligée. Il est essentiel de comprendre la relation entre l'utilisation de la contraception (y compris la contraception d'urgence), les grossesses non désirées et les interruptions volontaires de grossesse pour briser le cycle des avortements à répétition, mais nous ne disposons pas encore de rapports de terrain permettant de le faire. Il faudrait entreprendre des recherches qualitatives sur ces questions, en particulier des études pouvant mener à des interventions dans le cadre des programmes, compte tenu des perceptions des femmes et de leurs besoins. Une autre série de thèmes a trait au réseau social de la femme, et en particulier son mari ou son partenaire. Certains projets commencent à explorer la participation masculine et ces premiers efforts ont obtenu des résultats prometteurs. Ces études doivent être élargies à d'autres situations afin de mieux connaître les moyens d'intégrer les hommes, dans le respect des normes déontologiques, aux soins post-abortum prodigués à leurs épouses.

La participation d'autres membres du réseau social de la femme aux soins post-abortum dépend de l'identification d'indicateurs appropriés de réussite permettant de mesurer l'impact du programme. Nous n'avons qu'une expérience limitée des études de suivi à long terme, qui reflètent partiellement la nature de la question. Il convient également de prendre en compte un certain nombre de questions déontologiques graves lors du suivi des patientes post-abortum, que des études longitudinales doivent résoudre. Bien que les études sur la réduction de la mortalité liée à l'avortement sortent du cadre des recherches opérationnelles, il convient d'enquêter sur l'impact des services post-abortum sur la limitation des avortements à répétition et sur l'utilisation durable de la contraception.

Au cours des cinq prochaines années, la recherche opérationnelle devra dépasser le stade des études pilotes et de démonstration. Il faut envisager d'élargir les stratégies et de reproduire les modèles qui ont été couronnés de succès. Le projet visant à établir une base de données mondiale, DATAPAC, va dans ce sens. L'établissement de partenariats entre des institutions assurant des prestations de services sanitaires et démographiques contribuera à l'évolution des soins post-abortum, qui quitteront le domaine de la recherche pour devenir des programmes à long terme. Pour assurer un stock constant d'instruments permettant de pratiquer l'évacuation par aspiration manuelle, il faudra s'allier à des entreprises du secteur privé, alors que la pression sur les donateurs pour qu'ils assurent les fonds nécessaires à la formation et à l'établissement de systèmes exige le soutien indéfectible du gouvernement. Le plaidoyer est un secteur important dans le domaine de la recherche opérationnelle et les conditions spéciales qui entourent les prestations de services post-abortum exigent la mise au point d'une stratégie de diffusion et de communication intelligente. Des ressources et une assistance technique suffisantes garantiront une bonne diffusion et une large utilisation des résultats des recherches opérationnelles post-abortum en cours, ce qui exige un attachement sans faille de la part des gouvernements et des donateurs aux objectifs de la Conférence internationale sur la population et le développement. Des progrès incroyables ont été accomplis dans le secteur des soins de santé de la reproduction destinés aux femmes. Le défi consiste aujourd'hui à tourner le dos à ce premier groupe d'études et à institutionnaliser des soins post-abortum sans danger et plus humains pour toutes les femmes qui en ont besoin. 


\section{Box page 1}

Les soins post-abortum sont par définition une stratégie de prestations de services reposant sur trois éléments :

- Services médicaux d'urgence (traitement des complications liées aux avortements spontanés ou provoqués dans des conditions dangereuses)

- Conseils, orientation et services de planification familiale post-abortum

- Liens avec d'autres services de soins de la reproduction. 


\section{Graphique 1}

\section{Chronologie des soins post-abortum}

1982 Ipas lance des projets pilotes pour le traitement des complications liées à l'avortement au Mexique

1984 La Conférence internationale des Nations Unies sur la population, tenue à Mexico, « gèle les travaux»

1985 L'OMS organise une Réunion internationale sur la prévention de la mortalité maternelle

1986 Le groupe de travail technique de l'OMS inscrit l'évacuation par aspiration (électrique et manuelle) sur la liste des procédures obstétriques essentielles

1987 Tenue à Nairobi d'une Conférence sur la maternité sans risques et sur l'amélioration de la santé des femmes et des enfants grâce à la planification familiale

1990 Ipas souligne l'importance de la planification familiale post-abortum. Conférence organisée à Tbilissi « De l'avortement à la contraception »

1991 Ipas lance l'initiative des soins post-abortum, qui est un volet critique de son plan stratégique

1992 «Meeting Women's Needs for Postabortion Family Planning: Framing the Questions », Issues in Abortion Care (Ipas)

1993 Conférence de Bellagio sur la planification familiale post-abortum (Ipas, Population Council, IPPF, South to South Co-operation in Reproductive Health); «Politique de Mexico» contournée, début du dégel; Ipas, Pathfinder International, AVSC International, IPPF, JHPIEGO créent le consortium des soins post-abortum; série de réunions à l'USAID; USAID souligne l'importance des soins post-abortum dans ses réunions.

1994 «Post-Abortion Care: A Women's Health Initiative to Combat Unsafe Abortion », Advances in Abortion Care (Ipas); USAID fait des soins post-abortum une priorité de la santé de la reproduction; le paragraphe 8.25 du Programme d'action de la Conférence internationale du Caire sur la population et le développement apporte un appui mondial aux soins post-abortum.

1995 La Conférence mondiale des Nations Unies sur les femmes tenue à Beijing fait une large place au problème de santé publique que représentent les avortements dangereux; la Conférence internationale des donateurs sur « la mise en œuvre des programmes de santé de la reproduction » souligne l'importance des soins post-abortum; l'OMS publie Complications of Abortion:

Technical and Managerial Guidelines for Prevention and Treatment; la recherche sur les soins post-abortum est incorporée aux projets INOPAL, RO Afrique, RO ANE et MotherCare

1996 Les soins post-abortum sont intégrés aux activités communautaires de santé de la reproduction et de planification familiale

1997 «Care for Postabortion Complications: Saving Women's Lives; publié dans la série Population Reports

Source : Greenslade et al. 


\section{Graphique 2}

Cadre pour des soins post-abortum de qualité

Informations et conseils

Interaction entre les femmes et le prestataires de services

Accès des femmes aux services

Lien avec les soins

Planification familiale

Traitement des complications post-abortum liées à l'avortement

Équipements, matériel et médicaments

Performance technique

Technologies appropriées pour le traitement des complications

Source : Greenslade et al. 
Graphique 3

Admission dans le service d'OB/GYN pendant les 30 jours qu'a duré l'étude sur les hôpitaux du secteur public en Égypte

Gynécologie 21\%

Obstétrique $60 \%$

Post-abortum 19\%

Type d'admission en OB/GYN

$(n=22$ 656)

Avortement certainement provoqué 5\%

Probablement provoqué $2 \%$

Peut-être provoqué $58 \%$

Spontané 35\%

Classification de l'OMS

( $n=4153$ patientes post-abortum)

Source : Huntington et al. 


\section{Graphique 4}

Efficacité des modèles de prestations de services

Pourcentage de femmes ayant accès à des conseils et à des méthodes de planification familiale, Kenya

Femmes ayant reçu des conseils sur la PF

Femmes ayant reçu une méthode de PF

$\%$

Modèle 1 : (personnel des services OB/GYN

Modèle 2 : (personnel responsable de la PF dans le service)

Modèle 3 : (clinique de PF)

Source : Solo et al. 


\section{Box page 8}

Atelier sur les relations personnelles organisé dans le cadre de l'étude des soins post-abortum réalisée à Oaxaca, Mexique

Pour sensibiliser le personnel hospitalier aux besoins des femmes ayant subi un avortement, tous les prestataires des services d'obstétrique et de gynécologie de l'hôpital a participé à un atelier de 14 heures sur les relations personnelles. Les objectifs de cet atelier étaient les suivants :

- Améliorer la qualité des soins administrés aux patientes post-abortum

- Favoriser un changement d'attitudes chez le personnel soignant afin de rendre les prestations plus humaines

- Expliquer que la qualité des soins et la chaleur humaine sont des aspects importants des soins intégrés

- Ne pas oublier qu'il y a une femme derrière chaque patiente et l'accompagner pendant la période de convalescence

- Mettre les compétences des professionnels de la santé au service de leur vocation.

Chacun de ces thèmes a été traité dans le cadre de discussions interactives, jeux de rôles, méditation, exercices de relaxation et techniques faisant appel à l'image.

Source : Langer et al., p. 12. 


\section{Graphique 5}

Pourcentage de femmes acceptant une méthode de contraception moderne, Bolivie

$\%$

Santa Cruz

La Paz

Sucre

2e semestre de 1995

1er semestre de 1996

2e semestre de 1996

1er semestre de 1997

Source : Diaz. 


\section{Graphique 6}

Conseils et services fournis en matière de planification familiale post-abortum, Pérou

Information sur la PF

Méthode de PF fournie

Info sur le réapprovisionnement

Orientation vers les services de PF

$\%$

Avant l'intervention Après l'intervention

Extrait des entretiens à la sortie de l'hôpital et des formulaires cliniques.

Source : Benson et al. 


\section{Graphique 7}

\section{Douleurs ressenties par les femmes lors des soins post-abortum, Mexico}

Pas de douleurs

Douleurs faibles

Douleurs modérées

Douleurs aiguës

$\%$

Modèle 1

(Évacuation par aspiration manuelle, anesthésie locale et conseils)

Modèle 2

(D et C, sédatifs puissants, conseils)

Modèle 3

(D et C, sédatifs puissants, pas de conseils)

$\mathrm{B}=$ avant traitement

$\mathrm{D}=$ durant le traitement

$\mathrm{A}=$ après traitement

$\mathrm{E}=$ entretien à la sortie

Source : Fuentes et al. 
Box page 15

«Au cours des quelques dernières années, quelques institutions turques ont appliqué avec succès des programmes pilotes destinés à améliorer la prestation des services de planification familiale et les conseils fournis aux clientes ayant subi un avortement. En liant les services d'avortement et périnatals aux services existants de planification familiale, en renforçant les compétences du personnel pour qu'il puisse donner des informations et des conseils de qualité, et utilisant tous les moyens permettant à l'homme d'utiliser et d'appuyer la contraception, ces établissements ont réussi à améliorer les conditions de vie des hommes et des femmes qu'ils desservent. Ces institutions se caractérisent toutes par l'engagement ou la «volonté politique» de leur personnel et de l'administration d'assurer des services de planification familiale postabortum.»

Source : Pile et al., p. 29. 


\title{
Graphique 8
}

Cadre pour l'introduction d'améliorations aux programmes de soins post-abortum

\section{PERSPECTIVES DE LA CLIENTE}

\author{
Besoins et droits \\ Expérience de la cliente \\ Profil médical \\ Influence socioculturelle et sexospécifique
}

\section{SYSTÈME DE SERVICES}

Politiques

générale

Structure du programme

Qualité des soins

Approvisionnement en matériel

Personnel, établissements, gestion

Disponibilité et accessibilité

Politique, Société, Économie

Source : Girvin et Pollack.

\section{TECHNOLOGIE}

Aspiration sans anesthésie 
Box page 28

Quatre principes de conduite déontologique

1) Comme la santé de la reproduction est un bien social important, promouvoir la santé de la reproduction et améliorer les conditions permettant de l'atteindre sont des obligations déontologiques.

2) La justice en matière de santé de la reproduction s'appuie sur un partage équitable des avantages et des responsabilités liés aux décisions en matière de reproduction, notamment la décision d'avoir ou non des enfants.

3) Les actions et pratiques déontologiques sont celles qui traitent les individus avec respect et respectent leur autonomie.

4) Les programmes et politiques de santé de la reproduction respectueux de la déontologie sont ceux dans lesquels les conséquences désirables prennent le pas sur les conséquences indésirables.

Source : Ringheim (extrait de «la Déclaration de principes déontologiques »). 


\section{Documents présentés à la réunion}

Pour obtenir des exemplaires de ces documents, s'adresser directement à l'auteur principal (se référer à la liste des participants pour toutes les informations nécessaires).

Abdel-Tawab, Nahla, Dale Huntington, Ezzeldin Osman Hassan, Hala Youssef et Laila Nawar, «Effects of husband involvement on postabortion patients' recovery and use of contraception in Egypt »

Ankrah, Victor, Kathlyn Ababio, Deborah Billings, Joseph Taylor, Traci Baird et Steve Ntow, «Community midwives in Ghana offer comprehensive postabortion care »

Benson, Janie, « Future directions in postabortion care research »

Benson, Janie, Victor Huapaya, Marian Abernathy et John Naghata, «Provider practices and patient perspectives in an integrated postabortion care model in Peru »

Billings, Deborah, Xochitl Castaneda Camey, Xochitl Romero, Virginia Chambers, Andrea Saldana, Hector M. Alvarez Valero, Julia Blanco et Patricia Voorduin, « Traditional midwives and postabortion care services in Morelos, Mexico »

Brambila, Carlos, Cecila Garcia-Barrios et Angela M. Heimburger, «Estimating costs of postabortion services at Aurelio Valdivieso General Hospital, Oaxaca, Mexico »

Diaz, Juan, Mariel Loayza, Yamile Torres de Yépez, Oscar Lora, Fernando Alvarez et Virginia Camacho, «Evaluation of a strategy for improving the quality of services and contraceptive acceptance in the postabortion period in three public-sector hospitals in Bolivia »

French, Bertha, Njoroge Waithaka et Achola Ominde, « Management of incomplete abortion in Kenya by clinical officers: A pilot study »

Fuentes Velazquez, Jaime A., Deborah L. Billings et Jorge Arturo Cardona Perez, «Women experience of pain during the postabortion period in Mexico »

Ghosh, Anita, Bibiane Kone, Jean Lankoande et Placide Tapsoba, «Introducing improved postabortion care into maternity services in Burkina Faso »

Greenslade, Forrest C., Judith Winkler et Ann H. Leonard, «A framework for evaluating the quality of postabortion care services »

Huntington, Dale et Laila Nawar, « Introducing improved postabortion care in Egypt: Moving from a pilot study to large-scale expansion »

Huntington, Dale, Laila Nawar, Ezzeldin Osman Hassan, Hala Youssef et Nahla Abdel-Tawab, «A descriptive study of the postabortion caseload in Egyptian hospitals »

Johnson, Brooke, Michael T. Mbizvo, Tsungai Chipato et Felicity Zawaira, « Conducting follow-up with postabortion women in Zimbabwe: Some preliminary descriptive findings »

Khan, M. E., S. Rajagopal, Sandhya Barge et Nayan Kumar, « Situation analysis of medical termination of pregnancy (MTP) services in Gujarat, Maharashtra, Tamil Nadu, and Uttar Pradesh »

King, Tim, Janie Benson et Karen Stein, « Comparing the cost of postabortion care in Africa and Latin America: The DATAPAC Project»

Langer, Ana, Cecilia Garcia-Barrios, Angela Heimburger, Lourdes Campero, Olivia Ortiz, Carmen Diaz, Vilma Barahona, Francisca Ramirez et Beatriz Casas, «Improving postabortion care in a public hospital in Oaxaca, Mexico »

Nawar, Laila, Dale Huntington et Mohammed Naguib, «Cost-effectiveness of postabortion services in Egypt » 
Pile, John, Arzum G. Çiloglu, Levent Çagatay et Çidgem Bumin, « The quality of abortion services in Turkey »

Ringheim, Karin, «Toward an understanding of ethical issues in postabortion care research - A background discussion paper»

Solo, Julie, Achola Ominde, Margaret Makumi, Deborah Billings et Colette Aloo-Obunga, «Creating linkages between incomplete abortion treatment and family planning services in Kenya: What works best? »

Stein, Karen, «Postabortion care: Standardizing instruments and the postabortion care database » 
Nahla Abdel-Tawab

Liste des participants

Country Fellow

Population Council

6 A Bahieddin Barakat Street, Giza

P.O. Box 115, Dokki 12211, le Caire, Égypte

Tél. : 202-573-8277/570-1733/571-9252

Téléc. : 202-570-1804

Courriel : ntawab@pccairo.org

\section{Marian Abernathy}

Program Associate for Latin America

Ipas

303 E. Main Street, P.O. Box 999

Carrboro, NC 27510

Tél. : 919-967-7052

Téléc. : 919-929-0258

Courriel : mariana@ipas.org

Victor Ankrah

Ministry of Health/Eastern Region

P.O. Box 175

Koforidua, Ghana

Tél. : 233-81-23351

Téléc. : 233-81-23351

Courriel : vankrah@africaonline.com.gh

\section{Ian Askew}

Project Director, Africa OR/TA

Population Council

Multichoice Towers, Upper Hill

P.O. Box 17643, Nairobi, Kenya

Tél. : 254-2-713480

Téléc. : 254-2-713479

Courriel : iaskew@popcouncil.or.ke

\section{Karen Beattie}

Program Manager for Research

AVSC International

79 Madison Avenue

New York, NY 10016

Tél. : 212-561-8458

Téléc. : 212-779-9439

Courriel : kbeattie@avsc.org

\section{Janie Benson}

Director, Health Systems Research

Ipas

303 E. Main Street, P.O. Box 999

Carrboro, NC 27510

Tél. : 919-967-7052

Téléc. : 919-929-0258

Courriel : bensonj@ipas.org 


\section{Deborah Billings}

Research Associate

Ipas

303 E. Main Street, P.O. Box 999

Carrboro, NC 27510

Tél. : 919-967-7052

Téléc. : 919-929-0258

Courriel : debbieb@ipas.org

\section{Carlos Brambila}

Associate

Population Council

Escondida 110, Col Villa Coyoacan

Mexico, DF 04000, Mexique

Tél. : 525-554-0388, 554-8610

Téléc. : 525-554-1226

Courriel : cbrambila@laneta.apc.org

\section{George Brown}

Vice-Président

Population Council

One Dag Hammarskjold Plaza

New York, NY 10017

Tél. : 212-339-0500

Téléc. : 212-755-6052

Courriel : gbrown@popcouncil.org

\section{Levent Çagatay}

Program Associate

AVSC International

Abidin Daver S. 7/6, Cankaya 06550

Ankara, Turquie

Tél. : 90-312-4411761

Téléc. : 90-312-4414674

Courriel : lcagatay@avsc.org.tr

\section{Margaret Catley-Carlson}

Présidente

Population Council

One Dag Hammarskjold Plaza

New York, NY 10017

Tél. : 212-339-0500

Téléc. : 212-755-6052

Courriel : mcatleycarlson@popcouncil.org

\section{Richard Columbia}

Director of Evaluation

Pathfinder International

9 Galen Street

Watertown, MA 02172

Tél. : 617-924-7200

Téléc. : 617-924-3833

\section{Kurus Coyaji}

Director and Honorary Professor 
KEM Hospital

Rasta Peth

Pune, 411 011, Inde

Tél. : 91-212-625-600

Téléc. : 91-212-625-603

Courriel : kurus.coyaji@sm4.sprintrpg.ems.vsnl.net.in

\title{
Juan Diaz
}

Senior Associate

Population Council

Caixa Postal 6168, 13081-970

Campinas, Sao Paulo, Brésil

Tél. : 55-19-2392856

Téléc. : 55-19-2392440

Courriel : pcbrazil@turing.unicamp.br

\section{Ousseynou Faye}

CEFROEP

Clinique Gynéco-obstétrique

Hôpital A, Le Dantec,

Dakar, Sénégal

\section{Michelle Folsom}

Regional ARH Advisor

USAID REDSO/ESA

P.O. Box 30261

Nairobi, Kenya

Tél. : 254-2-751613 ext. 2459

Téléc. : 254-2-743204

Courriel : mfolsom@usaid.gov

\section{James Foreit}

Project Director, INOPAL

Population Council

4301 Connecticut Ave. NW

Suite 280

Washington, DC 20008

Tél. : 202-237-9400

Téléc. : 202-237-8410

Courriel : jforeit@pcdc.org

\section{Bertha French}

Program Associate

Ipas

303 E. Main Street, P.O. Box 999

Carrboro, NC 27510

Tél. : 919-967-7052

Téléc. : 919-929-0258

Courriel : frenchbm@ipas.org

\author{
Alison Friedman \\ Research Associate \\ Ipas \\ 303 E. Main Street, P.O. Box 999 \\ Carrboro, NC 27510
}


Tél. : 919-967-7052

Téléc. : 919-929-0258

Courriel : friedman@ipas.org

\section{Jaime Fuentes}

Mexican Social Security Institute

Mier y Pesado \#120

Colonia de Valle

Mexico, D.F. 03100, Mexique

Tél. : 525-536-8861

Téléc. : 525-536-8861

\section{Cecilia Garcia-Barrios}

Consultant

Population Council

Escondida 110, Col Villa Coyoacan

Mexico, D.F., 04000, Mexique

Tél. : 525-598-7556

Téléc. : 525-554-1226

Courriel : inopalmix@laneta.apc.org

\section{Phyllis Gestrin}

Senior Advisor in Reproductive Health

Africa Bureau, USAID

1111 North 19th Street, Room 300

Rosslyn, VA 22209

Tél. : 703-235-4446

Téléc. : 703-235-4466

Courriel : pgestrin@usaid.gov

\section{Anita Ghosh}

Program Development Officer

JHPIEGO

Brown's Wharf

1615 Thames Street, Suite 200

Baltimore, MD 21231

Tél. : 410-955-4413

Téléc. : 410-955-6199

Courriel : anita@wpo.jhpiego.org

\section{Sally Girvin}

Medical Associate, PAC Program Manager

AVSC International

79 Madison Avenue

New York, NY 10016

Tél. : 212-561-8458

Téléc. : 212-779-9439

Courriel : sgirvin@avsc.org

\section{Joanne Gleason}

Program Manager, Africa OR/TA

Population Council

One Dag Hammarskjold Plaza

New York, NY 10017

Tél. : 212-339-0500 
Téléc. : 212-755-6052

Courriel : jgleason@popcouncil.org

Forrest Greenslade

President

Ipas

303 E. Main Street, P.O. Box 999

Carrboro, NC 27510

Tél. : 919-967-7052

Téléc. : 919-929-0258

Courriel : forrestg@ipas.org

Gustavo Gutierrez

Guatemalan Social Security Institute

c/o Population Council

8a Calle 14-44, Zona 13

Guatemala City, 01013 C.A., Guatemala

Tél. : 502-3-32-38-48 / 32-23-65

Téléc. : 502-3-62-72-27

Courriel : popcouncil@guate.net

\title{
Nicole Haberland
}

Program Manager

Gender, Family, and Development

Population Council

One Dag Hammarskjold Plaza

New York, NY 10017

Tél. : 212-339-0500

Téléc. : 212-755-6052

Courriel : nhaberland@popcouncil.org

Ezzeldin Osman Hassan

Executive Director

Egyptian Fertility Care Centre

2A El Mahrouki St. 7th floor

Mohandiseen, le Caire, Égypte

Tél. : 202-347-0674

Téléc. 202-346-8782

\author{
Angela Heimburger \\ Consultant \\ Population Council \\ Escondida 110, Col Villa Coyoacan \\ Mexico, D.F. 04000, Mexique \\ Tél. : 525-554-0388 \\ Téléc. : 525-554-1226 \\ Courriel : pcmexico@popcouncil.org
}

\section{Marge Horn}

Office of Population

USAID/G/A\&D/POP

SA 18 Room 809

Washington, DC 20523-1819

Tél. : 202-712-4757

Courriel : mhorn@usaid.gov 


\section{Victor Huapaya}

Population Council

Paseo Padre Constancio Bollar 225

El Olivar de San Isidro L-27

Lima, Pérou

Tél. : 511-442-04-48

Téléc. : 511-440-06-35

Courriel : postmast@pclima.org.pc

Dale Huntington

Senior Program Associate

Population Council

6A Bahieddin Barakat Street, Giza

P.O. Box 115, Dokki 12211,

Le Caire, Égypte

Tél. : 202-573-8277/570-1733/571-9252

Téléc. : 202-570-1804

Courriel : dhunt@pccairo.org

\section{Anrudh Jain}

Director of Programs

Population Council

One Dag Hammarskjold Plaza

New York, NY 10017

Tél. : 212-339-0500

Téléc. : 212-755-6052

Courriel : ajain@popcouncil.org

\section{Barbara Janowitz}

Family Health International

P.O. Box 13950

Research Triangle Park, NC 27709

Tél. : 919-544-7040

Téléc. : 703-516-9781

\section{Brooke Johnson}

Deputy Director, Health Systems Research Ipas

303 E. Main Street, P.O. Box 999

Carrboro, NC 27510

Tél. : 919-967-7052

Téléc. : 919-929-0258

Courriel : rjohnson@ipas.org

\section{E. Khan}

Program Associate and Country Advisor

Population Council

53 Lodi Estate

New Delhi, 110003, Inde

Tél. : 91-11-461-0914/13

Téléc. : 91-11-461-0912

Courriel : pcnewdelhi@popcouncil.org 


\section{Tim King}

Research Associate

Ipas

303 E. Main Street, P.O. Box 999

Carrboro, NC 27510

Tél. : 919-967-7052 ext. 339

Téléc. : 919-929-0258

Courriel : king@ipas.org

\section{Jean Lankoanade}

CRESAR

Clinique Gynéco-obstétrique

Hôpital Yalago BP 4759

Ouagadougou, Burkina Faso

Tél. : 226-380419

Téléc. : 226-380419

Courriel : jlankoande@minitel.refer.org

\section{Robert Leke}

Associate Professor

Université de Yaoundé

BP 1935

Yaoundé, Cameroon

Tél. : 237-22-36-17

Téléc. : 237-23-44-51

\section{Noel McIntosh}

President

JHPIEGO

Brown Wharf

1615 Thames Street, Suite 200

Baltimore, MD 21231

Tél . : 410-955-8558

Téléc. : 410-955-6199

Courriel : nmc@jhpiego.org

\section{Esther Muia}

Associate, Reproductive Health Program

Population Council

Multichoice Towers, Upper Hill

P.O. Box 17643, Nairobi, Kenya

Tél. : 254-2-713480

Téléc. : 254-2-71-3479

Courriel : emuia@popcouncil.or.ke

\section{Donna Nager}

Program Manager, ANE OR/TA

Population Council

One Dag Hammarskjold Plaza

New York, NY 10017

Tél. : 212-339-0500

Téléc. : 212-755-6052

Courriel : dnager@popcouncil.org 


\section{Laila Nawar}

Host Country Advisor

Population Council

6A Bahieddin Barakat Street, Giza

P.O. Box 115, Dokki 12211,

Le Caire, Égypte

Tél. : 202-570-1733

Téléc. : 202-570-1804

Courriel : lnawar@pccairo.org

\section{Achola Ominde}

MCH/FP Manager

Division of Primary Health Care

P.O. Box 43319

Nairobi, Kenya

Tél. : 254-2-716812

Téléc. : 254-2-716814

\section{Emma Ottolenghi}

Consultant

Population Council

P.O. Box 308

Waterbury Center, VT 05677

Tél. : 802-244-8407

Téléc. : 802-244-8302

Courriel : emmao@igc.org

\section{Amy Pollack}

President

AVSC International

79 Madison Avenue

New York, NY 10016

Tél. : 212-561-8458

Téléc. : 212-779-9439

Courriel : apollack@avsc.org

\section{DM. Hafizur Rahman}

Institute of Research for Promotion of Essential and Reproductive Health (BIRPERHT)

House \#195, Road 9A

Dhanmondi R/A Dhaka-1209

Bangladesh, GPO Box 279

Tél. : 880-2-911-0792

Téléc. : 880-2-911-3034

Courriel : birperht@citechco.net

\section{Karin Ringheim}

Senior Technical Advisor, Operations Research USAID

G/PHN/POP/R

1300 Pennsylvania Avenue

Washington, DC 20523-3061

Tél. : 202-712-4758

Courriel : kringheim@usaid.gov 


\author{
Khama Rogo \\ Center for the Study of Adolescence \\ Kabarnet Road \\ Nairobi, Kenya \\ Tél. : 254-56-29-01 \\ Téléc. : 254-57-02-54 \\ Julie Solo \\ Staff Program Associate \\ Population Council \\ Multichoice Towers, Upper Hill \\ P.O. Box 17643 \\ Nairobi, Kenya \\ Tél. : 254-2-713480 \\ Téléc. :: 254-2-713479 \\ Courriel : jsolo@popcouncil.or.ke

\section{Karen Stein} \\ Progam Associate, International Programs Division \\ Population Council \\ One Dag Hammarskjold Plaza \\ New York, NY 10017 \\ Tél. : 212-339-0500 \\ Téléc. : 212-755-6052 \\ Courriel : kstein@popcouncil.org

\section{Placide Tapsoba} \\ Program Associate, Africa OR/TA Project \\ Population Council \\ Villa Nx4 Stele Mermoz \\ Route de la Pyrotechnie \\ BP 21207 \\ Dakar, Sénégal \\ Tél. : 221-241-993/4 \\ Téléc. : 221-241-998 \\ Courriel : pcdakar@sonatel.senet.net
}

\title{
Kathryn Tolbert
}

Program Associate

Population Council

Escondida 110, Col Villa Coyoacan

Mexico, D.F. 04000, Mexique

Tél. : 525-554-0388

Téléc. : 525-554-1226

Courriel : ktolbert@laneta.apc.org

\section{Andy Thompson}

Research Associate

Family Health International

Nairobi, Kenya

Tél. : 254-2-713-480

Téléc. : 254-2-213-479

Courriel : athompson@popcouncil.or.ke 


\author{
John Townsend \\ Project Director, ANE OR/TA \\ Population Council \\ 53 Lodi Estate \\ New Delhi, 110003, Inde \\ Tél. : 91-11-461-0914/13 \\ Téléc. : 91-11-461-0912 \\ Courriel : pcindiareg@popcouncil.org \\ Sandra Waldman \\ Director, Office of Public Information \\ Population Council \\ One Dag Hammarskjold Plaza \\ New York, NY 10017 \\ Tél. : 212-339-0500 \\ Téléc. : 212-755-6052 \\ Courriel : swaldman@popcouncil.org

\section{Nancy Williamson} \\ Family Health International \\ P.O. Box 13950 \\ Research Triangle Park, NC 27709 \\ Tél. : 919-544-7040 \\ Téléc. : 703-516-9781

\section{Beverly Winikoff} \\ Director, Reproductive Health Program \\ Population Council \\ One Dag Hammarskjold Plaza \\ New York, NY 10017 \\ Tél.: 212-339-0500 \\ Téléc. : 212-755-6052 \\ Courriel : bwinikoff@popcouncil.org
}

